\title{
44. PALYNOLOGICAL ANALYSIS OF DSDP LEG 42B (1975) CORES FROM THE BLACK SEA
}

\author{
Alfred Traverse, The Pennsylvania State University, College of Earth and Mineral Sciences, \\ University Park, Pennsylvania
}

\section{INTRODUCTION}

Initial palynological study of the Black Sea sediments from DSDP Leg 42B (1975), Sites 379, 380, and 381 , is based on investigation of core-catcher samples, about 250 in all. The spacing of the core-catcher units, approximately 10 meters apart, is too wide for the study to provide more than very broad answers to the many questions with which palynology should be expected to help regarding chronology, paleoclimatology, paleoecology, and stratigraphy. Study of more closely spaced samples is underway. The Black Sea sediments contain numerous palynomorphs for marine sediments; practically every sample contains them, both spore/pollen and phytoplankton.

Site reports elsewhere within this volume present a major aspect of the palynological results, that is the "steppe-index" and "marine-influence-index" information for the three sites. The curves obtained by these two indices suggest revelation of important facts about climatic and sedimentary history of the Black Sea basin; the reader is directed to the site chapters for plots of the index data.

\section{MATERIALS AND METHODS}

Core-catcher samples studied were mostly silty clays with varying amounts of carbonate. Most samples were unconsolidated, although a few indurated samples, including some limestones, were encountered. During shipboard work, prolonged heating in $20 \% \mathrm{HCl}$, followed by boiling with Calgon detergent, and gravity separation with $\mathrm{ZnCl}_{2}$ solution (specific gravity 2.0 ) produced satisfactory preparations from nearly all samples, but substitution on shore of $52 \%$ HF digestion for detergent dispersion resulted in more concentrated and cleaner preparations.

\section{SYSTEMATIC COMMENTS}

\section{Pollen and Spores}

Most of the identifiable palynomorphs that were recovered are referable to genera (Figures 1-3 and Tables 1-3). Pinus and Cedrus are combined because of difficulties in separating them in routine analysis; presumably most of the counts are of Pinus. Similarly, Abies and Picea are combined and most of the counts are probably Abies. In addition to these four, other kinds of bisaccate conifer pollen occur. For the most part these are presented here as undifferentiated bisaccates, although future work may enable more precise identification. The "bisaccate problem" is intensified by the occurrence, at some levels, of obviously reworked Mesozoic and Paleogene conifer pollen.

"Mesozoic-Paleogene triporates" (Tables 1-3) include a variety of Normapolles and post-Normapolles. Similarly, "trilete spores, undifferentiated include many Mesozoic-Paleogene reworked spores (most of which are identifiable), as well as spores presumably referable to extant genera.

\section{Acritarchs}

This category of palynomorphs includes forms of uncertain relationship. As their identity is established, acritarchs are classified with other groups, for example, as dinoflagellates when tabulation, archeopylesopercula, and/or other characteristic dinoflagellate structures are observed. "Dinoflagellate 19-20", was originally considered to be a pollen grain, then an acritarch until good archeopyles and opercula were observed. Another form (fungal $10=$ acritarch 10a), originally classified as an acritarch, later was determined to be a fungal spore. Still other acritarchous forms could conceivably turn out to be dinoflagellates, such as acritarch-13 (quite possible, as some likely dinoflagellate archeopyles were seen) and acritarch-8 (less likely, but not impossible). Although one "acritarchous" form turned out to be a fungal spore, the overwhelming majority of them can be assumed to be part of the life cycle of a marine alga-that is, some sort of phytoplankton. Thus the calculation of "marine-influence indices" from the combined totals of dinoflagellates and acritarchs (Figure 5, Site 379; Figure 5, Site 380; Figure 4, Site 381) is an acceptable procedure. Plate 1, Figures 1-4, and 12-17, show some of the characteristic acritarch types from the Black Sea cores.

\section{Dinoflagellates}

Fossil dinoflagellates are, in nearly all instances, the resistant-walled encysted stages of the life cycle of this group of one-celled organisms. Most fossil cysts are of marine origin, although fossil fresh-water dinoflagellate cysts are known. Because much of the history of the Black Sea represented by our cores was that of a fresh or brackish lake, dinoflagellates do not make up as large a part of the fossil record as they would in a truly marine sequence. Nevertheless, they are sufficiently abundant, at certain levels, to represent marine incursions.

Also, as mentioned above, a number of nondescript, "baggy" forms turned out to be undoubted although rather bizarre dinoflagellates, such as dinoflagellates $19,20,24 a$, and 29 . These "baggy" cysts are probably brackish water and specialized forms that flourished 


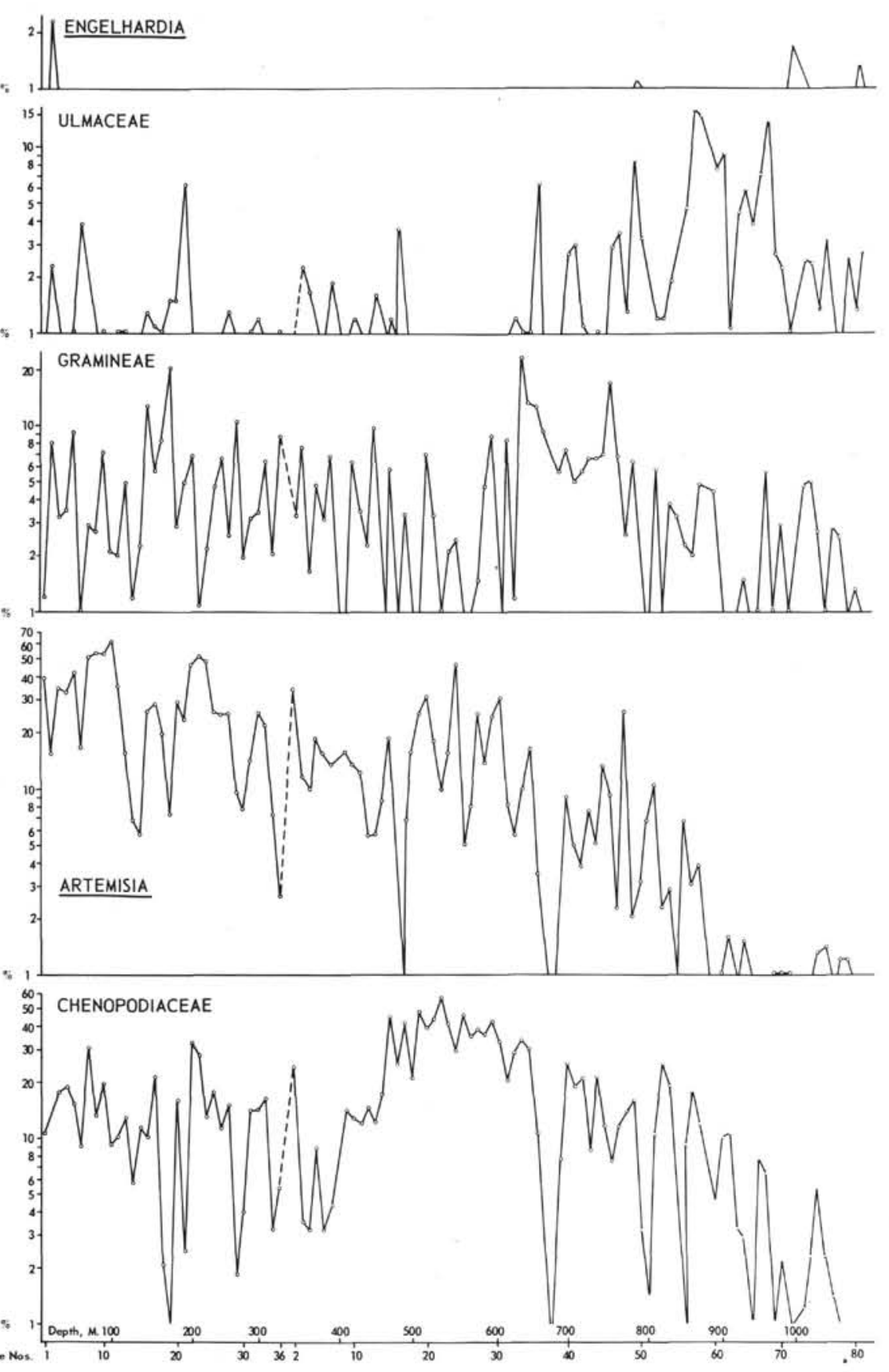

Figure 1. Pollen distribution, major types, Holes 380/380A, core-catcher samples, plotted semi logarithmically. The small circles represent cores. Where the curve intersects the bottom line with no circle showing, a number less than one is indicated. Circles on the baseline indicate a percentage of one. As explained in the site reports, Core 37 of Hole 380 is correlated palynologically with Core 1 of Hole $380 \mathrm{~A}$. 

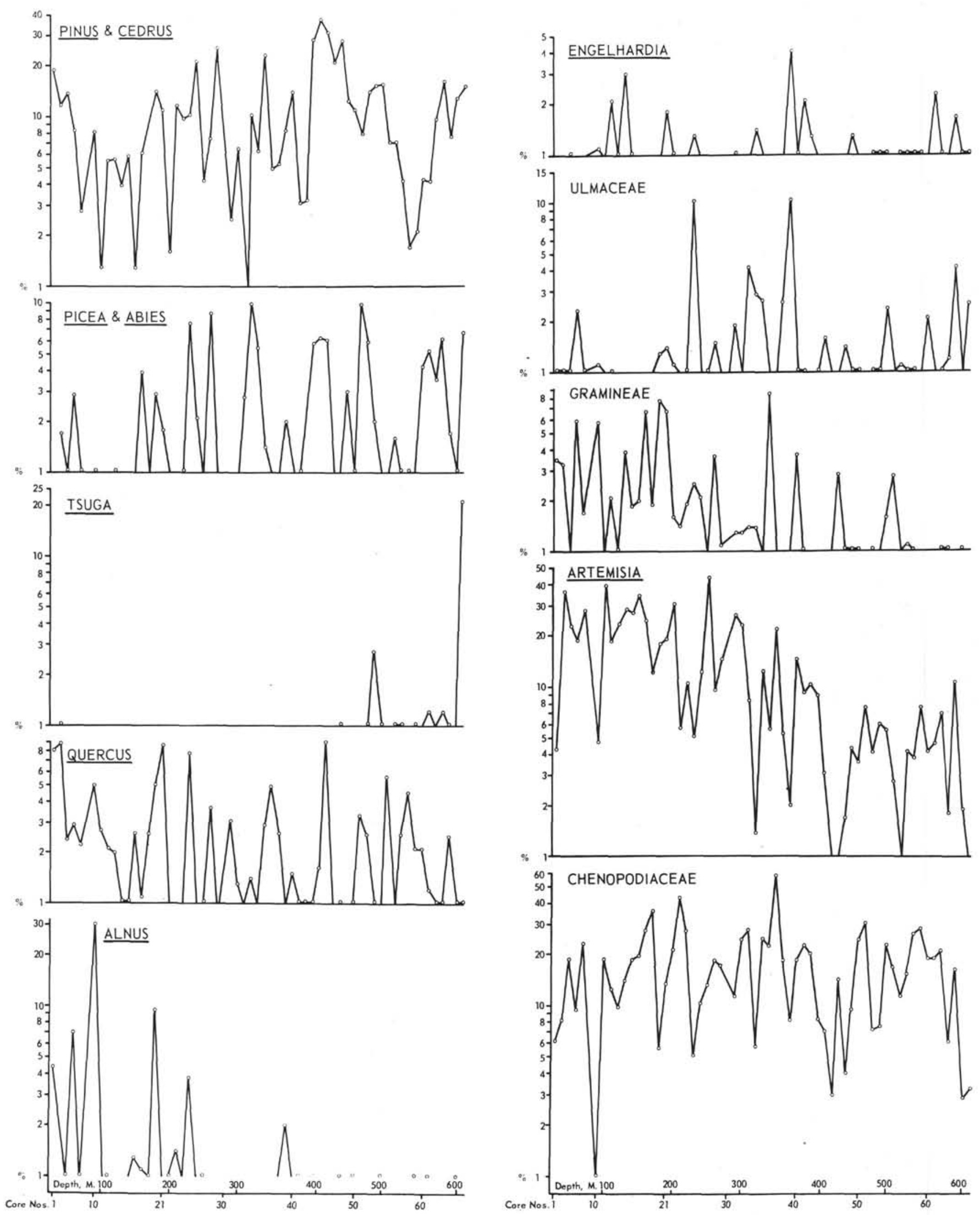

Figure 2. Pollen distribution, major types, Hole 379, core-catcher samples, plotted as explained in the caption to Figure 1. 

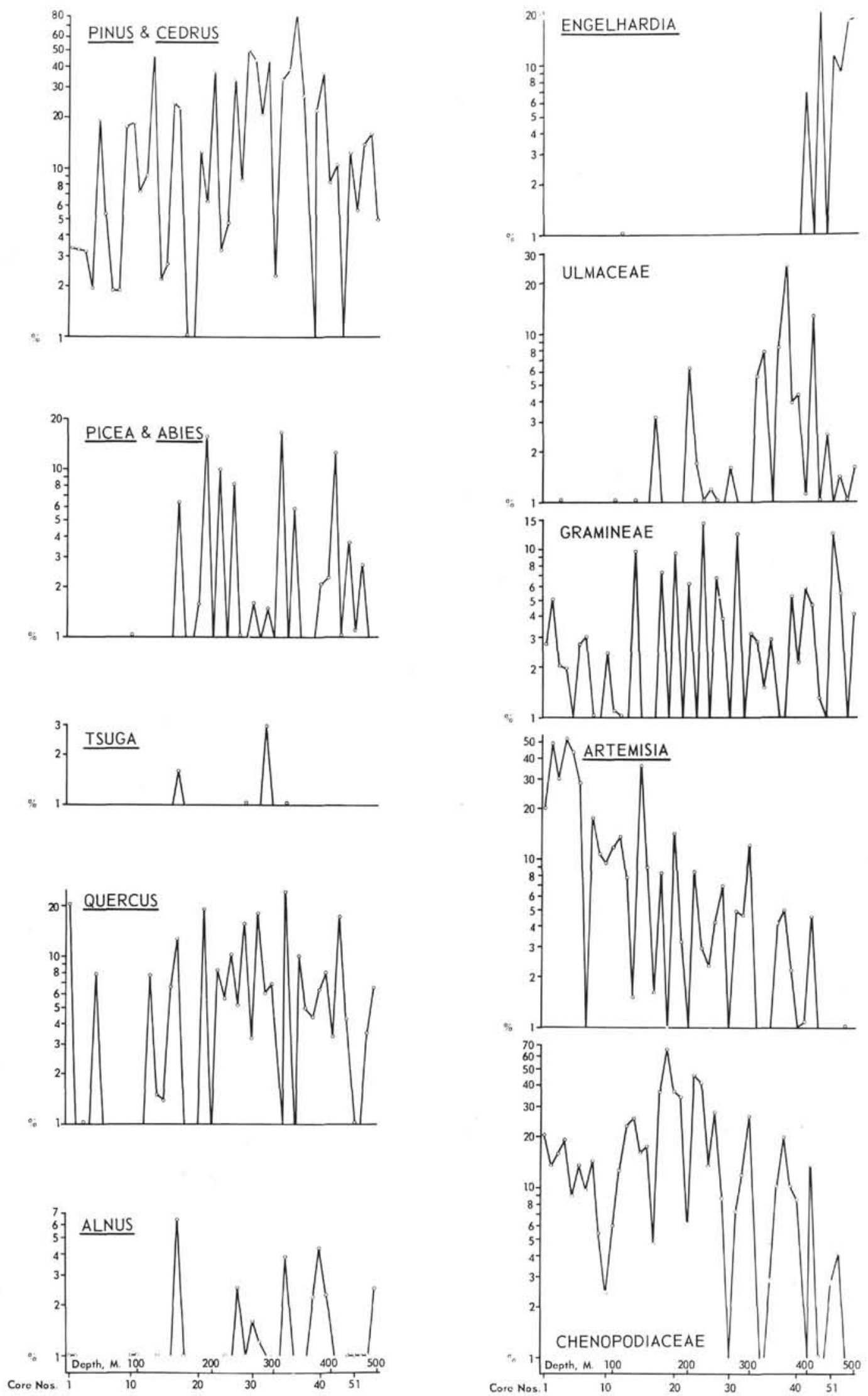

Figure 3. Pollen distribution, major types, Hole 381, core-catcher samples, plotted as explained in the caption to Figure 1. 
when the water freshened. They tend to be present locally in great abundance as in the case of dinoflagellate-29 which makes up almost $100 \%$ of the microfossils found in Sample 381-38, CC (Table 3); a single slide contains at least 4000 specimens! This sort of occurrence must bespeak of an unusual set of ecological circumstances.

\section{Fungal Spores}

As can be seen from Tables 1-3, fungal spores and fruiting bodies are a regular constituent of the palynoflora of the Leg 42B cores. Fragments of fungal mycelia are also common, particularly in samples containing a large amount of organic debris. Fungal-10 and fungal-11, which are the dominant elements in some samples are, according to W.C. Elsik who studied my slides, possibly phycomycetes. From my experience it is rare for fungal spores to be so overwhelmingly dominant in a marine or near-marine sediment as these forms are in certain samples (see, for example, Sample $381-35$, CC, where the 4000 fungal spores counted are practically all fungal-11).

\section{PALYNOMORPH DISTRIBUTION}

Figures 1-3 show graphically the distribution of major components of the palynoflora of the Black Sea sediments. The sample spacing, one per core, is too wide (approximately one per $10 \mathrm{~m}$ ) to follow minor fluctuations in climate, but broad trends are shown. Summary figures from the Site Chapters representing the same samples should be used in conjunction with the present Figures 1-3. However, the Site Chapter curves are drawn on the basis of a running average. The present curves are straight plots of the data on semilogarithmic paper, to accentuate the smaller percentages. Although these graphs are based on widely spaced samples, they do show that floral changes, reflecting climatic and other environmental factors, were dramatic and frequent during the part of the Neogene sampled, as would be expected (see van der Hammen et al., 1971). The curves in Figures 1-3 show distribution of only a relatively few palynomorph types: Pinus + Cedrus (mostly Pinus), Picea + Abies (mostly Abies), Tsuga, Quercus, Alnus, Engelhardia, Ulmaceae, Gramineae, Artemisia, and Chenopodiaceae. These and all other counts obtained in this study are presented in Tables 1-3.

\section{Holes 380/380A}

These holes (Figure 1) are the standard for the other two because the record is more nearly complete; Hole 381 probably penetrates older sediments.

Age: The much higher percentages of total conifers from about Hole $380 \mathrm{~A}$, Core 35 down probably represents the preglacial part of the Pleistocene. Tsuga is particularly important in this regard. Small Tsuga counts (2\%-3\%) in two of the higher samples are somewhat puzzling, because Tsuga is not likely to be reworked, being insufficiently resistant, but Tsuga pollen is present in practically all samples from Core 32 downwards. This fits well with the greater abundance of Ulmaceae pollen below Core 38. The almost complete absence of Artemisia pollen below Core 60 probably represents the Pliocene-Pleistocene interface; in applied palynology in the American Gulf Coast, such an advent of Artemisia would be interpreted as the onset of a colder, drier, Pleistocene climate. The much lower values of Chenopodiaceae in this part of the record is coordinated with the Artemisia data. As noted in the Site Chapters, presumed palm pollen (monocolpate, reticulate) occurs from Core 71 downward. This probably represents early Pliocene sediment. The counts of Engelhardia in a few samples below Core 70 may be of reworked specimens, but if they are not, it would indicate that the cores at this level are late Miocene (van der Hammen et al., 1971), or earliest Pliocene in age (Planderová and Gabrielová, 1975). There is no question, however, that at Site 381 older sediments than at Site 380 are penetrated.

\section{Site 379}

Although the curves presented in the Site Chapters, based on running averages, present a fairly clean picture, the curves shown here (Figure 2) are somewhat difficult to interpret, in part because there is obvious reworking. A good example of this is Engelhardia, all counts of which are presumably reworked pollen. The relatively high Tsuga counts at the bottom of Site 379, in Core 68, probably do correlate with similar counts beginning about Core 32 of Hole $380 \mathrm{~A}$. Tsuga pollen is not as likely to survive reworking as well as Engelhardia pollen, and the Tsuga counts probably do not represent reworked pollen to a significant extent. The Artemisia curve shows the same decline as seen in the lower part of the 380/380A record. As noted in the Site Chapters, based on correlations of the "SFI" and "MI" the bottom of Site 379 probably is not older than Waalian, that is, about mid-Quaternary.

\section{Site 381}

The curves for Site 381 are shown in Figure 3. As noted in the Site Chapters, the record appears to be interrupted, representing at most two of the cold periods ("Alpha" and "Gamma"). The Tsuga counts, while not many or large, show the existence of the preglacial Quaternary from about Core 15 downward, which ties in well with abundant ulmaceous pollen and general decline of Artemisia. As also noted earlier, the abundant Engelhardia pollen below Core 43 indicates early Pliocene or late Miocene age, and the lowest part of the record, which also has fairly abundant palm pollen (monocolpate, reticulate) and a number of early Neogene forms such as various "Tricolpites" spp. (for example, " $T$. microhenricii") indicates that the bottom of Site 381 is well into Miocene rocks (van der Hammen et al., 1971; Planderová and Gabrielová, 1975).

\section{SUMMARY AND CONCLUSIONS}

If the information from the marine-influence ("MI") curves and steppe-influence ("SI") curves are combined with the percentage curves and total count information provided here, a reasonable overall picture of the palynological analysis of the Black Sea cores is obtained, but more detailed analyses from more closely 
TABLE 1

Palynological Analyses, Site 379

\begin{tabular}{|c|c|c|c|c|c|c|c|c|c|c|c|c|c|c|c|c|c|}
\hline & 1 & 4 & 6 & 7 & 8 & 9 & 10 & 11 & 12 & 13 & 14 & 15 & 16 & 17 & 18 & 19 & 21 \\
\hline Fungal spores & 1 & 3 & 2 & 13 & 1 & & 5 & 6 & 7 & 11 & 10 & 1 & 26 & 6 & 33 & 14 & 16 \\
\hline \multicolumn{18}{|l|}{ Dinoflagellates } \\
\hline Undifferentiated & 1 & 3 & 3 & 8 & 1 & & - & 5 & - & 3 & 1 & 11 & 8 & 8 & 3 & 2 & 8 \\
\hline Dino-11 & - & - & - & 13 & - & & - & - & - & - & - & - & - & - & - & - & - \\
\hline Dino-14 & - & 24 & 1 & - & - & & - & - & - & - & - & - & - & - & - & - & - \\
\hline Dino-19\&20 & - & - & - & $4 ?$ & 1 & \pm & - & - & - & - & - & - & 3 & 1 & - & 1 & 1 \\
\hline Botryococcus & & & & & & B & & & & & & & & & & & \\
\hline Pediastrum & & & & & & A & & & & 1 & & & & 1 & & & \\
\hline Acritarchs & & & & & & $\mathrm{R}$ & & & & & & & & & & & \\
\hline Undifferentiated & 46 & 28 & 29 & 23 & 7 & $\mathrm{R}$ & 16 & 9 & 10 & 17 & 14 & 7 & 31 & 13 & 17 & 13 & 48 \\
\hline Ac- 8 & - & 31 & 10 & 6 & 1 & $\mathrm{E}$ & 3 & 3 & 9 & 3 & 4 & 8 & 15 & 1 & 1 & 7 & 2 \\
\hline Cymatiosphaera & - & - & - & - & - & $\mathrm{N}$ & - & - & - & - & - & - & - & - & - & - & - \\
\hline Tasmanites & - & - & - & - & - & & - & - & - & - & - & - & - & - & - & - & - \\
\hline "Circuli-" & 1 & - & - & - & - & & - & - & - & - & - & - & - & - & - & - & - \\
\hline \multicolumn{18}{|l|}{ Trilete spores } \\
\hline Undifferentiated & 2 & - & - & 1 & - & & - & - & - & - & - & 1 & 1 & - & - & 1 & 1 \\
\hline Cicatricosisporites, rew. & - & - & - & - & - & & - & 1 & - & - & - & - & - & - & - & - & - \\
\hline Klukisporites & - & - & - & - & - & & - & - & 1 & - & - & - & - & - & - & - & - \\
\hline Sphagnum & - & - & - & - & - & & - & - & - & - & - & - & - & - & - & - & - \\
\hline Monolete spores & - & - & - & - & - & & 2 & - & - & - & - & - & - & $\bar{x}$ & - & 1 & 2 \\
\hline Undetermined pollen & 48 & 35 & 66 & 48 & 51 & 1 & 41 & 42 & 67 & 87 & 45 & 35 & 42 & 36 & 51 & 89 & 36 \\
\hline "Tetrad-1" & 1 & - & - & - & - & & - & - & - & - & - & - & - & - & - & - & 1 \\
\hline Monocolpate & - & - & 1 & 1 & - & - & - & - & - & 3 & - & - & - & 1 & - & 3 & 3 \\
\hline Mesozoic-Paleogene triporates & - & - & 1 & - & - & - & 1 & - & 1 & - & 1 & - & - & - & - & - & 1 \\
\hline \multicolumn{18}{|l|}{ Conifers-Gymnosperms } \\
\hline Bisaccate & - & 6 & - & - & - & - & 3 & - & - & 3 & 3 & 3 & 1 & 1 & - & 1 & 7 \\
\hline Inaperturate & - & 1 & - & - & - & - & 2 & - & 2 & 7 & 1 & 1 & - & - & 7 & 7 & 2 \\
\hline Cedrus & - & 6 & 4 & - & 1 & - & 1 & - & 1 & 2 & - & - & - & 1 & - & 2 & 1 \\
\hline Picea-Abies & - & 3 & 2 & 5 & 1 & - & 2 & - & - & 1 & - & - & - & 7 & - & 11 & 4 \\
\hline Pinus & 21 & 15 & 24 & 14 & 4 & - & 21 & 2 & 7 & 9 & 5 & 6 & 2 & 10 & 1 & 51 & 23 \\
\hline Sciadopitys & - & - & - & - & - & - & - & - & - & - & - & - & - & - & - & - & - \\
\hline Sequoia & - & - & - & - & - & - & - & - & - & - & - & - & - & - & - & - & - \\
\hline Tsuga & - & 1 & - & - & - & - & - & - & - & $-\cdot$ & - & - & - & - & - & - & - \\
\hline Vitreisporites & - & - & - & - & - & - & - & - & - & - & 1 & - & - & - & - & - & - \\
\hline Corollina & - & - & - & - & - & - & - & - & - & - & - & - & - & - & - & - & - \\
\hline Ephedra & - & 1 & - & - & - & - & - & - & - & 2 & - & 1 & 1 & - & - & - & 1 \\
\hline \multicolumn{18}{|l|}{ Monocots } \\
\hline Cyperaceae & 1 & - & - & 1 & - & - & - & - & - & - & - & - & - & - & - & 3 & - \\
\hline Gramineae & 4 & 6 & - & 10 & 3 & - & 16 & - & 3 & 2 & 5 & 2 & 3 & 12 & 3 & 30 & 15 \\
\hline Palmae & - & - & - & - & - & - & - & - & - & - & - & - & - & - & - & - & - \\
\hline Typha & - & - & - & - & - & - & - & - & - & - & - & - & - & - & - & - & - \\
\hline \multicolumn{18}{|l|}{ Dicots } \\
\hline Alnus & 5 & - & 1 & 12 & 1 & 1 & 84 & - & 1 & - & - & - & 2 & 2 & 1 & 36 & - \\
\hline Amaranthaceae & - & 1 & 2 & 1 & 2 & - & - & 4 & 2 & 1 & 1 & - & 4 & 3 & 2 & - & 2 \\
\hline Betula & - & - & - & - & 1 & - & - & 3 & - & - & - & - & - & 1 & 1 & 2 & 3 \\
\hline Carpinus - Ostrya & 5 & 2 & 1 & 5 & 4 & - & 14 & 1 & 2 & - & 1 & - & - & 1 & - & 5 & - \\
\hline Carya & - & 1 & - & - & - & - & - & 1 & - & - & - & 1 & 1 & 1 & - & - & 2 \\
\hline Castanea & - & - & - & - & - & - & - & - & - & - & - & - & - & - & - & - & - \\
\hline Celtis & - & - & - & - & 1 & - & - & - & - & - & - & - & - & - & - & 1 & - \\
\hline \multirow{2}{*}{\multicolumn{18}{|c|}{ Compositae }} \\
\hline & & & & & & & & & & & & & & & & & \\
\hline Undifferentiated & - & - & - & - & - & - & - & - & - & - & - & - & - & - & - & - & - \\
\hline Artemisia & 5 & 66 & 46 & 32 & 50 & - & 13 & 59 & 27 & 47 & 37 & 28 & 53 & 44 & 19 & 69 & 43 \\
\hline Long-spined & - & 3 & 7 & 5 & 5 & - & - & 3 & 3 & 7 & 3 & 2 & 5 & 2 & 2 & 5 & 9 \\
\hline Fenestrate & - & - & - & - & 2 & - & 1 & - & 1 & 1 & - & - & 1 & - & - & - & 1 \\
\hline Corylus & 1 & - & 1 & - & - & - & 4 & 1 & - & - & 1 & 1 & - & 1 & 3 & 1 & - \\
\hline Engelhardia & - & - & 1 & - & - & - & 3 & - & 2 & 2 & 4 & 1 & - & - & - & - & 4 \\
\hline Ericaceae & - & - & - & - & - & - & - & - & - & - & - & - & - & - & - & - & - \\
\hline Fagus & 1 & - & 1 & 6 & 5 & 5 & 42 & - & 2 & - & 1 & - & - & 3 & 5 & 12 & 5 \\
\hline Fraxinus & - & - & - & - & - & - & - & - & - & - & - & - & - & - & - & - & - \\
\hline Myrica & - & - & - & 2 & - & - & 1 & - & - & - & - & - & - & - & - & 1 & 1 \\
\hline Nyssa & - & - & - & - & - & - & - & - & - & - & - & - & - & - & - & - & - \\
\hline Pterocarya & - & 1 & 2 & 3 & 2 & - & 1 & - & - & - & - & - & - & 2 & 1 & 3 & 2 \\
\hline Quercus & 9 & 16 & 5 & 5 & 4 & - & 14 & 4 & 2 & 4 & 1 & 1 & 4 & 2 & 4 & 19 & 19 \\
\hline Tilia & - & - & - & - & - & - & 2 & - & 1 & - & 1 & 1 & - & - & - & - & - \\
\hline Ulmaceae & 1 & 1 & 1 & 4 & 1 & - & 3 & - & 1 & - & - & - & - & - & - & 5 & 3 \\
\hline Umbelliferae & - & - & - & - & - & - & - & - & - & - & - & - & - & - & - & - & - \\
\hline Zelkova & - & - & - & - & - & - & - & - & 1 & - & - & - & - & - & - & - & 1 \\
\hline
\end{tabular}


TABLE 1 - Continued

\begin{tabular}{|c|c|c|c|c|c|c|c|c|c|c|c|c|c|c|c|c|c|c|c|c|}
\hline 22 & 23 & 24 & 25 & 26 & 27 & 28 & 29 & 30 & 31 & 32 & 33 & 34 & 35 & 36 & 37 & 38 & 39 & 40 & 41 & 42 \\
\hline 13 & - & 12 & 1 & - & 6 & - & 6 & & 1 & - & - & - & - & - & 2 & 2 & 1 & - & 2 & 4 \\
\hline 1 & - & - & - & - & 7 & 1 & - & & 1 & 4 & - & - & - & - & - & - & - & - & 2 & - \\
\hline - & - & - & - & - & - & - & - & \pm & - & - & - & - & - & - & - & - & - & - & - & - \\
\hline- & - & - & - & - & - & - & - & B & - & - & - & - & - & - & - & - & - & - & - & - \\
\hline- & - & - & - & - & 30 & 6 & - & A & - & - & - & - & - & 40 & 450 & - & 1 & - & - & - \\
\hline- & - & - & - & - & - & - & - & $\mathrm{R}$ & - & - & 1 & - & - & - & - & - & - & - & - & - \\
\hline - & - & - & - & - & - & - & - & $\mathrm{R}$ & - & - & - & - & - & - & - & - & - & - & - & - \\
\hline 13 & 6 & 40 & 28 & 40 & 11 & 16 & 6 & $\mathrm{~N}$ & 8 & 20 & 15 & 10 & 15 & 12 & - & 2 & 5 & 7 & 18 & 9 \\
\hline 1 & - & - & - & - & 2 & - & - & & 6 & - & - & - & - & - & - & - & 1 & 4 & 2 & - \\
\hline- & 1 & - & - & - & - & - & - & & - & - & - & - & - & - & - & - & - & - & - & - \\
\hline- & - & - & - & - & - & - & - & 1 & - & - & - & - & - & - & - & - & 1 & - & - & - \\
\hline- & - & - & - & - & - & - & - & & - & - & - & - & - & - & - & - & - & - & - & - \\
\hline 1 & - & 1 & 1 & - & - & - & - & 1 & - & - & 4 & - & - & 5 & - & - & - & - & 1 & 1 \\
\hline - & - & - & - & - & - & - & - & & - & - & - & - & - & - & - & - & - & - & - & - \\
\hline- & - & - & - & - & - & - & - & & - & - & - & - & - & - & - & - & - & - & - & - \\
\hline- & - & - & - & - & - & - & - & & - & - & - & - & - & - & - & - & - & - & - & - \\
\hline- & - & - & - & 1 & 1 & 2 & 1 & & - & - & 3 & 2 & - & 2 & - & 1 & 1 & 1 & 1 & 2 \\
\hline 67 & 20 & 40 & 22 & 20 & 35 & 54 & 30 & 1 & 67 & 29 & 22 & 26 & 37 & 14 & 2 & 20 & 25 & 36 & 81 & 76 \\
\hline- & - & - & - & - & - & - & - & & 1 & - & - & - & - & - & - & - & - & - & - & - \\
\hline- & - & - & 1 & - & 1 & - & - & - & 1 & - & - & - & - & 1 & - & - & - & - & - & - \\
\hline 1 & - & 2 & 1 & - & - & - & 1 & - & 3 & 1 & - & - & - & - & 1 & - & 1 & - & 3 & - \\
\hline 7 & - & - & 4 & 1 & - & 4 & 2 & - & 2 & - & 2 & 10 & 2 & - & - & 1 & 3 & 9 & 17 & 9 \\
\hline 1 & - & - & - & - & - & - & - & - & 4 & - & 1 & 1 & - & - & - & $\cdot 1$ & - & - & 5 & 1 \\
\hline- & - & - & - & - & - & - & - & - & - & - & - & - & - & - & - & - & - & 1 & 1 & - \\
\hline- & - & 1 & 6 & 1 & - & 12 & - & 4 & - & - & 2 & 7 & 6 & 1 & - & - & 1 & - & 1 & - \\
\hline 3 & 8 & 10 & 8 & 10 & 5 & 10 & 22 & - & 4 & 5 & - & 7 & 7 & 16 & 2 & 2 & 4 & 17 & 5 & 5 \\
\hline- & 2 & - & - & - & - & - & - & - & - & - & - & 1 & - & - & - & - & - & - & - & - \\
\hline- & - & - & - & - & - & - & - & - & - & - & - & - & - & - & - & - & - & - & - & - \\
\hline - & - & - & - & - & - & - & - & - & - & - & - & - & - & - & - & - & - & - & - & - \\
\hline - & - & - & - & - & - & - & - & & & - & - & - & - & - & - & - & - & - & - & - \\
\hline - & - & - & - & - & - & - & 1 & - & - & - & - & - & - & - & - & - & - & - & - & - \\
\hline- & - & - & - & - & 1 & - & 1 & - & - & 1 & 1 & - & - & - & - & - & - & 3 & - & 3 \\
\hline - & - & - & 1 & - & - & - & - & - & - & - & _- & - & - & - & - & - & - & - & - & - \\
\hline 3 & 1 & 2 & 2 & 1 & - & 5 & 1 & - & 2 & 1 & 1 & 1 & - & 6 & - & - & - & 5 & 2 & - \\
\hline- & - & - & - & - & - & - & - & - & - & - & - & - & - & - & - & - & - & - & - & - \\
\hline- & - & - & - & - & - & - & - & - & - & - & - & - & - & - & - & - & - & - & - & - \\
\hline 1 & 1 & - & 3 & - & 1 & - & - & - & - & - & - & - & - & - & - & - & 1 & - & 1 & - \\
\hline- & 1 & 1 & - & - & 2 & 2 & 1 & 1 & - & - & - & 1 & - & 1 & 1 & 1 & - & 2 & 4 & 4 \\
\hline- & - & 1 & - & - & - & - & - & - & - & - & - & 1 & - & - & - & - & 1 & 1 & - & - \\
\hline- & - & 1 & 4 & 1 & - & 1 & - & - & - & - & 1 & - & 1 & - & - & 1 & - & 2 & - & - \\
\hline - & - & - & - & - & - & - & - & - & - & - & - & 1 & - & - & - & - & - & - & - & - \\
\hline- & - & - & - & 1 & - & - & - & - & - & - & - & - & - & - & - & - & - & - & - & - \\
\hline- & - & - & - & - & - & - & - & - & - & - & - & - & - & - & - & - & - & - & - & - \\
\hline 41 & 30 & 29 & 4 & 5 & 16 & 25 & 15 & - & 18 & 19 & 20 & 4 & 28 & 16 & 24 & 7 & 4 & 24 & 44 & 31 \\
\hline- & 3 & 4 & 1 & 1 & - & - & - & - & - & 3 & 3 & 1 & 2 & 1 & - & - & - & - & - & - \\
\hline 61 & 4 & 11 & 4 & 6 & 53 & 13 & 13 & - & 43 & 18 & 6 & 1 & 14 & 4 & 9 & 2 & 1 & 19 & 18 & 16 \\
\hline 4 & - & - & - & - & 3 & - & - & - & 5 & - & - & - & - & - & - & - & - & 6 & 4 & 2 \\
\hline- & - & - & - & - & - & - & - & - & - & - & - & - & - & - & - & - & - & - & - & - \\
\hline 1 & - & - & 1 & - & - & - & - & - & - & - & 1 & - & 2 & - & - & - & - & - & - & - \\
\hline 1 & - & - & 1 & - & - & - & - & - & 1 & - & - & 1 & - & - & - & - & 2 & 1 & 4 & 2 \\
\hline - & - & - & - & - & - & - & - & - & - & - & 1 & - & - & - & - & - & - & - & - & - \\
\hline- & - & - & 1 & 1 & - & - & - & - & - & - & - & 2 & 1 & - & - & - & - & - & - & - \\
\hline - & - & - & - & - & - & - & - & - & - & - & - & - & - & - & - & - & - & - & - & - \\
\hline - & - & - & - & - & - & - & - & - & - & - & - & - & - & - & - & - & - & - & - & - \\
\hline - & - & - & - & - & - & - & - & - & - & - & - & - & - & - & - & - & - & - & - & - \\
\hline - & - & - & - & - & - & - & - & - & - & - & - & - & - & - & - & - & - & - & - & - \\
\hline- & - & - & 6 & - & 1 & 5 & - & - & 5 & 1 & - & 1 & - & 2 & 2 & 1 & - & 2 & 1 & 1 \\
\hline _ & - & - & - & - & - & - & - & - & - & - & - & - & - & 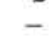 & 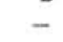 & - & - & - & - & 1 \\
\hline 2 & - & 1 & 8 & - & 1 & 2 & - & - & 3 & - & 3 & 2 & 3 & - & - & 1 & 5 & 1 & 1 & - \\
\hline - & - & - & - & - & - & - & - & - & - & - & - & - & - & 1 & - & - & - & - & - & - \\
\hline - & - & - & - & - & - & - & - & - & - & - & - & - & - & - & - & - & - & - & - & - \\
\hline
\end{tabular}




\begin{tabular}{|c|c|c|c|c|c|c|c|c|c|c|c|c|c|c|c|c|c|}
\hline & 43 & 45 & 46 & 47 & 48 & 49 & 50 & 51 & 52 & 53 & 54 & 55 & 56 & 57 & 58 & 59 & 60 \\
\hline Fungal spores & 2 & 1 & 1 & 1 & 4 & - & - & - & - & - & 2 & - & 5 & 5 & 5 & 12 & 1 \\
\hline \multicolumn{18}{|l|}{ Dinoflagellates } \\
\hline Undifferentiated & - & 1 & - & - & - & 6 & - & 4 & 2 & - & - & 1 & - & 1 & 7 & 6 & 19 \\
\hline Dino-11 & - & - & - & - & - & - & - & - & - & - & - & - & - & - & - & - & - \\
\hline Dino-14 & - & - & - & - & - & - & - & - & - & - & - & - & - & - & - & - & - \\
\hline Dino-19\&20 & 2 & - & - & - & 2 & - & 1 & 384 & 100 & 14 & - & 1 & 28 & 3 & 44 & 45 & 165 \\
\hline Botryococcus & - & 1 & 2 & - & - & - & 1 & - & - & - & 1 & - & - & - & - & - & - \\
\hline Pediastrum & - & - & - & - & 1 & - & - & - & - & - & - & - & - & - & - & - & - \\
\hline \multicolumn{18}{|l|}{ Acritarchs } \\
\hline Undifferentiated & 26 & 19 & 4 & 4 & 32 & 15 & 3 & 5 & 23 & 11 & 11 & 6 & 12 & 16 & 20 & 57 & 11 \\
\hline$A c-8$ & 4 & 7 & 4 & 1 & - & 5 & 4 & 1 & 3 & - & - & 1 & 5 & 2 & - & - & 2 \\
\hline Cymatiosphaera & - & - & - & - & - & - & - & - & - & - & - & - & - & - & - & - & - \\
\hline Tasmanites & - & - & - & - & - & - & - & - & - & - & - & - & - & - & - & - & - \\
\hline "Circuli-" & - & - & - & - & - & - & - & - & - & - & - & - & - & - & - & - & - \\
\hline \multicolumn{18}{|l|}{ Trilete spores } \\
\hline Undifferentiated & 2 & 1 & 1 & 1 & 6 & 1 & 4 & 1 & 6 & - & 1 & - & - & 3 & 2 & - & - \\
\hline Cicatricosisporites, rew. & - & - & - & - & - & - & - & - & 1 & - & - & _ & - & - & - & - & _- \\
\hline Klukisporites & - & - & - & - & - & - & - & - & - & - & - & - & - & - & - & - & - \\
\hline Sphagnum & - & - & - & - & - & - & - & - & 1 & - & - & - & - & - & - & - & 1 \\
\hline Monolete spores & 1 & - & - & - & 1 & - & 2 & - & 5 & 1 & 1 & - & 3 & 1 & - & 1 & - \\
\hline Undetermined pollen & 69 & 34 & 10 & 15 & 125 & 73 & 25 & 24 & 76 & 44 & 39 & 25 & 103 & 152 & 149 & 104 & 23 \\
\hline "Tetrad-1" & - & - & - & - & - & - & - & - & - & - & - & - & - & - & - & 1 & - \\
\hline Monocolpate & - & - & - & - & 1 & - & 1 & - & 2 & 1 & 1 & - & 1 & - & - & 2 & 2 \\
\hline \multirow{2}{*}{\multicolumn{18}{|c|}{ Conifers-Gymnosperms }} \\
\hline & & & & & & & & & & & & & & & & & \\
\hline Bisaccate & 12 & 15 & 2 & 2 & 25 & 62 & 13 & 9 & 46 & 40 & 20 & 13 & 20 & 19 & 7 & 18 & 3 \\
\hline Inaperturate & - & - & 1 & - & 9 & 5 & - & 1 & 3 & 2 & - & - & 3 & 1 & 4 & 13 & 1 \\
\hline Cedrus & - & 5 & 3 & 1 & 28 & 13 & 4 & - & 8 & 3 & 3 & 1 & 3 & 1 & 3 & - & 1 \\
\hline Picea-Abies & 11 & 8 & 2 & - & -. & 7 & 1 & 9 & 14 & 3 & - & - & 3 & 1 & 2 & 1 & 2 \\
\hline Pinus & 52 & 42 & 7 & 6 & 52 & 15 & 8 & 7 & 25 & 19 & 16 & 4 & 10 & 11 & 2 & 6 & 1 \\
\hline Sciadopitys & - & - & - & - & - & - & - & - & - & - & - & - & - & - & - & - & - \\
\hline Sequoia & - & - & - & - & 1 & 1 & - & - & 1 & - & - & - & - & - & - & - & - \\
\hline Tsuga & - & - & - & - & 1 & - & - & - & 1 & 4 & 1 & - & 1 & 1 & - & 2 & - \\
\hline Vitreisporites & - & - & - & - & - & - & - & - & 1 & - & - & - & - & - & - & - & - \\
\hline Corollina & - & - & - & - & 1 & - & - & - & - & - & - & - & - & - & - & - & - \\
\hline \multirow{2}{*}{\multicolumn{18}{|c|}{ Monocots }} \\
\hline & & & & & & & & & & & & & & & & & \\
\hline Cyperaceae & - & - & - & - & - & - & - & - & - & - & - & - & - & - & - & - & - \\
\hline Gramineae & - & - & - & 1 & 3 & 1 & 1 & - & 2 & - & 2 & 2 & - & 3 & 1 & - & - \\
\hline Palmae & - & - & - & - & - & - & - & - & - & - & - & - & - & - & - & - & - \\
\hline Typha & - & - & - & - & - & - & - & - & - & - & - & - & - & - & - & - & - \\
\hline \multicolumn{18}{|l|}{ Dicots } \\
\hline Alnus & 1 & - & - & - & 1 & - & 1 & - & - & - & 1 & - & - & - & - & 2 & - \\
\hline Amaranthaceae & - & 1 & - & 1 & - & 1 & 5 & - & - & - & - & 1 & 2 & 7 & 4 & 2 & - \\
\hline Betula & - & - & - & - & 1 & 2 & - & - & - & - & - & - & - & - & - & 1 & - \\
\hline Carpinus - Ostrya & 1 & 1 & 1 & 1 & 2 & 1 & - & - & - & - & - & - & 1 & 1 & - & - & - \\
\hline Carya & - & - & - & 1 & 1 & - & - & - & 1 & 1 & - & - & - & 1 & - & - & - \\
\hline Castanea & - & - & - & - & - & - & - & - & - & - & - & - & - & - & - & - & - \\
\hline Celtis & - & - & - & - & - & - & - & - & 1 & - & - & - & - & - & - & - & - \\
\hline Chenopodiaceae & 16 & 9 & 1 & 5 & 12 & 22 & 27 & 28 & 17 & 11 & 28 & 12 & 21 & 44 & 76 & 81 & 9 \\
\hline $\begin{array}{l}\text { Compositae } \\
\text { Undifferentiated }\end{array}$ & & & & & & & & & & & & & & & & & \\
\hline $\begin{array}{l}\text { Undufterentrated } \\
\text { Artemisia }\end{array}$ & 17 & 4 & - & - & 5 & 10 & 4 & $\overline{7}$ & 10 & 9 & 7 & 2 & - & $\overline{12}$ & $\overline{11}$ & $2 \overline{2}$ & 2 \\
\hline Long-spined & 3 & 3 & - & - & 7 & 10 & 7 & 1 & 2 & 2 & - & 3 & 5 & 8 & 4 & 14 & 2 \\
\hline Fenestr: & - & - & - & - & 1 & 3 & - & 1 & 1 & - & - & 3 & 2 & 2 & - & 1 & - \\
\hline Corylus & - & - & - & - & - & - & - & - & - & - & - & - & - & - & - & - & - \\
\hline Engelhardia & - & - & - & - & - & 3 & - & - & 2 & 1 & 1 & - & 1 & 2 & 3 & - & - \\
\hline Ericaceae & - & - & - & - & - & - & - & - & - & - & - & - & - & - & - & - & - \\
\hline Fagus & - & - & - & - & - & - & - & - & - & - & - & - & - & - & - & - & - \\
\hline Fraxinus & 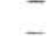 & - & - & - & - & - & - & _- & - & - & - & - & - & - & - & - & - \\
\hline Myrica & - & - & 1 & - & 2 & - & - & - & 1 & - & - & 1 & 1 & - & - & - & - \\
\hline Nyssa & - & - & - & - & 1 & - & - & - & - & - & - & - & - & - & - & - & - \\
\hline Pterocarya & - & - & _ & - & - & 1 & - & - & - & - & - & - & - & - & 1 & 1 & - \\
\hline Quercus & 1 & 2 & 3 & - & 2 & - & 1 & 3 & 6 & 1 & - & 4 & 1 & 7 & 13 & 6 & 1 \\
\hline Tilia & - & - & - & 1 & - & 1 & - & - & - & - & - & - & - & - & - & - & - \\
\hline Ulmaceae & 1 & 2 & - & - & 4 & 1 & 1 & - & 1 & 1 & 3 & - & 2 & 1 & 3 & - & 1 \\
\hline Umbelliferae & - & - & - & - & - & - & - & - & - & - & - & - & - & - & - & - & - \\
\hline Zelkova & - & - & - & - & - & - & - & - & - & - & - & - & - & - & - & - & - \\
\hline
\end{tabular}


TABLE 1 - Continued

\begin{tabular}{|c|c|c|c|c|c|}
\hline 62 & 63 & 64 & 65 & 66 & 68 \\
\hline 6 & 5 & 1 & 3 & 9 & 39 \\
\hline 8 & 4 & 8 & 3 & 15 & 4 \\
\hline- & - & - & - & - & - \\
\hline- & - & - & - & - & - \\
\hline 88 & 10 & 182 & 6 & 2 & 2 \\
\hline- & - & - & - & - & 2 \\
\hline- & - & - & - & - & - \\
\hline 26 & 14 & 20 & 21 & 59 & 28 \\
\hline 2 & - & 1 & 1 & 2 & 25 \\
\hline- & - & - & - & - & - \\
\hline- & - & - & 1 & - & - \\
\hline- & - & - & - & - & - \\
\hline 3 & 1 & 1 & 2 & 4 & 3 \\
\hline- & - & - & - & - & - \\
\hline- & 1 & - & - & - & - \\
\hline- & - & - & - & - & - \\
\hline 1 & 1 & 1 & 1 & 2 & 3 \\
\hline 59 & 38 & 31 & 33 & 61 & 55 \\
\hline- & - & - & - & - & 1 \\
\hline 4 & 2 & - & - & - & - \\
\hline 1 & - & - & 5 & 2 & - \\
\hline 29 & 14 & 62 & 11 & 75 & 50 \\
\hline 1 & - & - & 1 & 5 & 1 \\
\hline 1 & 6 & 3 & 1 & 1 & 23 \\
\hline 9 & 4 & 10 & 2 & 2 & 16 \\
\hline 6 & 5 & 23 & 8 & 25 & 13 \\
\hline- & - & - & - & - & - \\
\hline- & - & 1 & - & - & - \\
\hline 2 & - & 2 & 1 & 8 & 52 \\
\hline- & 1 & 2 & 2 & - & 1 \\
\hline- & - & - & - & - & - \\
\hline 1 & 2 & - & - & - & - \\
\hline- & - & - & - & - & - \\
\hline- & 1 & 1 & - & 2 & - \\
\hline- & - & - & - & - & - \\
\hline- & - & - & - & - & - \\
\hline 1 & - & - & - & 1 & - \\
\hline 1 & 2 & 1 & 1 & - & - \\
\hline - & - & - & - & - & 1 \\
\hline- & - & - & - & 1 & - \\
\hline 1 & - & - & 1 & 1 & 1 \\
\hline- & - & - & - & - & - \\
\hline- & - & - & - & - & - \\
\hline 33 & 24 & 10 & 20 & 6 & 8 \\
\hline- & - & - & - & - & - \\
\hline 8 & 8 & 3 & 13 & 4 & - \\
\hline 4 & 6 & 7 & 4 & 4 & 4 \\
\hline 1 & - & 1 & - & 2 & 1 \\
\hline- & - & - & 1 & - & - \\
\hline 4 & 1 & - & 2 & 2 & 2 \\
\hline- & - & - & - & - & - \\
\hline- & - & - & - & - & - \\
\hline- & - & - & - & - & - \\
\hline- & - & - & - & - & - \\
\hline- & - & - & - & - & - \\
\hline- & - & - & - & - & 2 \\
\hline 2 & 1 & 1 & 3 & 1 & 2 \\
\hline- & - & - & 1 & - & - \\
\hline- & 1 & 2 & 5 & - & 6 \\
\hline- & - & - & - & - & - \\
\hline- & - & - & 1 & - & - \\
\hline
\end{tabular}

spaced samples will be required to follow Neogene climatic and vegetational fluctuations, of which the Black Sea cores provide a unique record. The palynological study demonstrates the prevailingly fresh to brackish water conditions in the Black Sea basin during most of Neogene time, and the record also supports a picture of dramatically fluctuating climatic conditions during the Pleistocene with, however, an indication of a limited number of large-scale cold (probably three) and warm periods. One very significant point that emerges from the palynological analyses is that the apparent transgressive periods in Black Sea history were not all necessarily coordinated with the warmer periods, as might have been expected from the post-glacial record (see Traverse, 1971). Indeed, the most dramatic transgressive episode occurred during cold period "Alpha" (see Site chapters, especially pollen figure for Site $380)$.

\section{ACKNOWLEDGMENTS}

The assistance of Dr. D.M. McLean, Virginia Polytechnic Institute, and Dr. W.C. Elsik, Exxon Company, Houston, Texas, with identification of phytoplankton and fungal spore material, respectively, is gratefully acknowledged.

\section{REFERENCES}

Planderová, E. and Gabrielová, N., 1975. Biozones of Neogene stages from the viewpoint of micropaleobotany. In Cicha, I. (Ed.), Biozonal division of the Upper Tertiary basins of the eastern Alps and west Carpathians: VI Congress of Regional Committee on Mediterranean Neogene Stratigraphy: Prague (Bratislava. Geol. Survey), p. 101-109.

Traverse, A., 1974. Palynologic investigation of two Black Sea cores, in the Black Sea-geology, chemistry and biology: Am. Assoc. Petrol. Geol. Mem. 20, p. 381-388.

Traverse, A. and Ginsburg, R.N., 1966. Palynology of the surface sediments of Great Bahama Bank, as related to water movement and sedimentation: Marine Geol., v. 4, p. $417-459$.

van der Hammen, T., Wijmstra, T.A., and Zagwijn, W.H., 1971. The floral record of the late Cenozoic of Europe. In Turekian, K.K. (Ed.), The late Cenozoic glacial ages: New Haven (Yale Univ. Press), p. 391-424.

\section{PLATES 1 AND 2, ILLUSTRATING REPRESENTATIVE PHYTOPLANKTONIC AND FUNGAL FORMS}

General note: Most of the palynomorphs encountered in these cores are common, well-known forms of pollen and spores. It is really not necessary to illustrate or describe such well-known fossils as Artemisia and Alnus pollen grains and Sphagnum spores. However, a number of unusual fungal-spore and phytoplanktonic fossils do occur and are abundant and important in certain samples. These will be described and illustrated here, though the descriptions are brief and informal, and no formal nomenclature will be introduced at this time. All photos are $\pm 1000 \times$, except where otherwise noted. 
TABLE 2

Palynological Analyses, Holes 380/380A

\begin{tabular}{|c|c|c|c|c|c|c|c|c|c|c|c|c|c|c|c|c|c|c|c|c|}
\hline 380 & 1 & 2 & 4 & 5 & 6 & 7 & 8 & 9 & 10 & 11 & 12 & 13 & 14 & 15 & 16 & 17 & 18 & 19 & 20 & 21 \\
\hline Fungal spores & - & - & - & - & - & 1 & - & - & - & - & - & 2 & - & - & - & - & - & - & - & - \\
\hline Dinoflagellates & & & & & & & & & & & & & & & & & & & & \\
\hline Undifferentiated & 1 & 2 & - & - & - & - & - & - & - & - & - & 1 & - & 9 & - & - & - & - & 1 & 1 \\
\hline Dino-19\&20 & - & - & - & - & - & - & - & - & - & - & - & - & - & - & - & - & - & - & - & - \\
\hline Botryococcus & - & - & - & 2 & - & 2 & 14 & - & - & - & - & - & - & - & - & - & - & - & - & - \\
\hline Pediastrum & - & 2 & 1 & - & 1 & - & 1 & - & - & - & 2 & 1 & - & - & 2 & - & - & - & - & 1 \\
\hline Acritarchs & & & & & & & & & & & & & & & & & & & & \\
\hline Undifferentiated & 4 & 15 & 1 & 45 & 1 & 83 & 59 & 2 & - & 3 & 3 & 5 & 7 & - & 4 & 4 & 5 & 43 & 1 & 2 \\
\hline$A c-1$ & - & 1 & - & - & - & - & - & - & - & - & - & - & - & - & - & - & - & - & - & - \\
\hline Ac-8 & - & - & - & - & - & - & - & - & - & - & - & - & - & - & - & - & - & - & - & - \\
\hline Cymatiosphaera & - & 5 & - & 1 & - & 2 & - & - & - & - & - & - & 1 & - & - & - & - & 1 & - & - \\
\hline Tasmanites & - & - & - & - & - & - & - & - & - & - & - & - & - & - & - & - & - & - & - & - \\
\hline "Circuli" & - & - & - & - & - & - & - & - & - & - & - & - & - & - & - & - & - & - & - & - \\
\hline Trilete spores & & & & & & & & & & & & & & & & & & & & \\
\hline Undifferentiated & 1 & 2 & 1 & - & - & - & - & 3 & 1 & 2 & - & 9 & 8 & 2 & - & 2 & 1 & 2 & - & 2 \\
\hline Sphagnum & - & - & - & - & - & - & - & - & - & - & - & 2 & 1 & - & - & - & - & - & - & - \\
\hline Klukisporites & - & - & - & - & - & - & - & - & - & - & - & - & - & - & - & - & - & - & - & - \\
\hline Monolete spores & 1 & - & - & 1 & 1 & - & - & - & - & 1 & 4 & 3 & 5 & 1 & 2 & - & - & 1 & 1 & - \\
\hline Undetermined pollen & 16 & 19 & 1 & 5 & 25 & 27 & 6 & 17 & 6 & 4 & 11 & 9 & 8 & 3 & 3 & 11 & 12 & 6 & 8 & 11 \\
\hline "Tetrad-1" & - & - & - & - & - & - & - & - & - & - & - & - & - & - & - & - & - & - & - & - \\
\hline Monocolpate & - & - & - & - & - & - & - & - & - & - & - & - & - & - & - & - & - & - & - & - \\
\hline Mesozoic-Paleogene triporates & - & 3 & - & - & 2 & - & - & 1 & - & 1 & 1 & - & - & 1 & - & - & 1 & - & 1 & 3 \\
\hline Conifers-Gymnosperms & & & & & & & & & & & & & & & & & & & & \\
\hline Bisaccate & 1 & 4 & 2 & - & - & 1 & - & 2 & - & - & 3 & 25 & 16 & 13 & 2 & 2 & 1 & 3 & 2 & 9 \\
\hline Inaperturate & - & 1 & - & 2 & 1 & - & - & 3 & 1 & - & 1 & 2 & 2 & 2 & 2 & 2 & 3 & 5 & 3 & 1 \\
\hline Cedrus & - & - & - & - & - & - & - & - & - & - & - & - & - & - & - & - & - & - & - & - \\
\hline Keteleeria & - & - & - & - & - & - & - & - & - & - & - & - & - & - & - & - & - & - & - & - \\
\hline Picea-Abies & - & - & - & - & - & 1 & - & - & - & - & - & - & 4 & 7 & 3 & 2 & 1 & 4 & 2 & - \\
\hline Pinus & 9 & 12 & 17 & 6 & 3 & 32 & - & 6 & 7 & 9 & 26 & 21 & 24 & 21 & 10 & 12 & 38 & 18 & 11 & 20 \\
\hline Sciadopitys & - & - & - & - & - & - & - & - & - & - & - & - & - & - & - & - & - & 1 & - & - \\
\hline Sequoia & - & - & - & - & - & - & - & - & - & - & - & - & - & - & - & - & - & - & - & - \\
\hline Taxodiad & - & 1 & 1 & - & - & - & - & - & - & - & - & - & - & - & 2 & 1 & - & 3 & - & - \\
\hline Tsuga & - & 3 & - & - & - & - & - & - & - & - & - & - & - & - & - & - & - & - & - & - \\
\hline Vitreisporites & - & - & - & - & - & - & - & - & - & - & - & - & - & - & - & - & - & - & - & - \\
\hline Corollina & - & - & - & - & - & - & - & - & - & - & 1 & - & - & - & - & - & - & - & - & - \\
\hline Ephedra & - & - & 1 & - & - & 1 & 1 & - & - & - & - & - & - & - & 1 & 1 & - & - & - & - \\
\hline Monocots & & & & & & & & & & & & & & & & & & & & \\
\hline Cyperaceae & _ & - & - & _- & - & - & _- & - & - & - & - & - & - & - & - & - & - & - & - & - \\
\hline Gramineae & 1 & 7 & 2 & 3 & 12 & 1 & 2 & 3 & 7 & 2 & 2 & 6 & 1 & 2 & 9 & 5 & 8 & 14 & 2 & 4 \\
\hline Palmae & - & - & - & - & - & - & - & - & - & - & - & - & - & - & - & - & - & - & - & - \\
\hline Typha & - & - & - & - & 1 & 2 & - & - & - & - & - & 1 & - & 1 & - & - & - & - & - & - \\
\hline Dicots & & & & & & & & & & & & & & & & & & & & \\
\hline Alangium & - & - & - & - & - & - & - & - & - & - & - & - & - & - & - & - & - & - & - & - \\
\hline Alnus & 1 & 5 & 1 & 4 & 1 & 7 & - & - & - & 1 & - & 1 & - & 2 & 1 & - & 1 & 1 & - & 1 \\
\hline Amaranthaceae & - & - & - & - & I & - & 1 & 1 & - & - & - & - & - & 1 & - & 2 & - & - & - & - \\
\hline Betula & - & 1 & - & 1 & - & 3 & - & - & 2 & 1 & - & 1 & - & - & 2 & - & 1 & - & 1 & - \\
\hline Carpinus - Ostrya & 1 & - & 1 & 2 & 3 & 3 & - & - & - & - & - & 1 & - & 3 & - & - & 1 & 1 & - & - \\
\hline Carya & - & - & - & 1 & - & - & - & - & - & - & - & 1 & - & - & - & - & - & - & - & - \\
\hline Castanea & - & - & - & 1 & - & - & - & - & - & - & - & - & - & - & - & - & - & - & - & - \\
\hline Castanopsis & - & 1 & - & - & - & - & - & - & - & - & - & - & - & - & - & - & - & - & - & - \\
\hline Celtis & - & - & - & - & - & - & - & - & - & - & - & - & - & - & - & - & - & - & - & - \\
\hline Chenopodiaceae & 9 & - & 11 & 16 & 20 & 14 & 21 & 15 & 19 & 9 & 10 & 16 & 5 & 10 & 8 & 19 & 2 & - & 11 & 2 \\
\hline Compositae & & & & & & & & & & & & & & & & & & & & \\
\hline Undifferentiated & 4 & 2 & - & 1 & 2 & 1 & 3 & 1 & - & 3 & - & 1 & 1 & 2 & 4 & - & 3 & 2 & 2 & 3 \\
\hline Artemisia & 33 & 14 & 22 & 29 & 56 & 26 & 35 & 60 & 52 & 60 & 35 & 19 & 6 & 5 & 20 & 25 & 19 & 5 & 20 & 19 \\
\hline Long-spined & - & - & - & - & - & - & - & - & - & - & - & - & - & - & - & - & - & - & - & - \\
\hline Fenestrate & - & - & - & - & - & - & - & - & - & - & - & - & - & - & - & - & - & - & - & - \\
\hline Corylus & 1 & - & - & 3 & 1 & 1 & - & - & - & - & - & - & 1 & 2 & 2 & 1 & 1 & - & 1 & - \\
\hline Engelhardia & - & 2 & - & - & - & - & - & - & - & - & - & - & - & - & - & - & - & 1 & - & - \\
\hline Ericaceae & - & - & - & - & - & - & - & - & - & - & - & - & - & - & - & - & - & - & - & - \\
\hline Fagus & - & - & - & 1 & 1 & 1 & - & - & - & - & - & - & - & - & - & - & - & - & - & - \\
\hline Fraxinus & - & - & - & - & - & 1 & - & - & - & - & - & - & - & - & - & - & - & - & - & - \\
\hline Ilex & - & - & - & - & - & - & - & - & - & - & - & - & - & - & - & - & - & - & - & - \\
\hline Itea & - & - & - & - & - & - & - & - & - & - & - & - & - & - & - & - & - & - & - & - \\
\hline Labiatae & - & - & - & - & - & 1 & - & - & - & - & 1 & 1 & - & - & - & - & - & - & - & - \\
\hline Liquidambar & - & - & - & - & - & - & - & - & - & - & - & - & - & - & - & - & - & - & - & - \\
\hline Moraceae & - & - & - & - & - & - & - & - & - & - & - & - & - & - & - & - & - & - & - & - \\
\hline Myrica & - & 2 & - & - & - & - & - & - & - & - & - & - & 1 & 1 & - & - & - & - & - & - \\
\hline Nyssa & - & - & - & - & - & - & - & - & - & - & - & - & - & - & - & - & - & - & - & - \\
\hline Parthenocissus & 1 & - & - & - & - & - & - & - & - & - & - & - & - & - & - & - & 2 & - & - & - \\
\hline Pterocarya & - & - & - & - & - & - & - & - & - & - & 1 & - & - & - & - & - & - & - & - & - \\
\hline Quercus & 6 & 7 & 3 & 9 & - & 23 & - & - & 1 & 3 & 2 & 3 & 4 & 8 & 4 & 2 & 1 & - & 2 & - \\
\hline Salix & - & - & - & - & - & - & - & - & - & 1 & - & - & - & - & - & - & - & - & - & - \\
\hline Tilia & - & - & - & 1 & 1 & 2 & - & - & - & - & - & - & - & - & 1 & - & - & - & - & - \\
\hline Ulmaceae & - & 2 & - & - & 1 & 6 & - & - & 1 & - & 1 & 1 & - & - & 1 & 1 & 1 & 1 & 1 & 5 \\
\hline Umbelliferae & - & - & - & - & - & - & - & - & - & - & - & - & - & - & - & - & - & - & - & - \\
\hline Zelkova & - & - & - & - & - & - & - & - & - & - & - & - & - & - & - & - & - & - & - & - \\
\hline
\end{tabular}


TABLE 2 - Continued

\begin{tabular}{|c|c|c|c|c|c|c|c|c|c|c|c|c|c|c|c|c|c|}
\hline 22 & 23 & 24 & 25 & 26 & 27 & 28 & 29 & 30 & 31 & 32 & 33 & 35 & 36 & 37 & 38 & 39 & 4 \\
\hline 1 & 1 & 1 & - & & 1 & - & 1 & 1 & - & - & 1 & - & - & - & - & 2 & - \\
\hline - & - & - & - & & - & - & - & 1 & - & - & - & 1 & - & - & - & 2 & - \\
\hline - & - & - & - & & 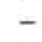 & - & - & - & - & 8 & 17 & - & 6 & - & 1 & 18 & \\
\hline - & - & $=$ & - & & 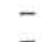 & - & - & - & - & 1 & 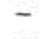 & - & - & - & 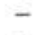 & - & \\
\hline- & - & - & - & B & - & & - & - & 4 & - & - & - & - & - & - & - & \\
\hline 1 & 2 & 3 & 2 & A & 3 & 2 & 4 & 1 & 10 & 3 & 39 & 6 & 102 & - & 2 & 13 & - \\
\hline - & - & - & - & $\begin{array}{l}R \\
D\end{array}$ & - & - & - & - & - & - & 1 & - & - & - & - & - & \\
\hline - & $\bar{z}$ & & - & $\mathrm{R}$ & $\overline{-}$ & - & - & - & - & - & - & 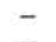 & - & - & - & - & - \\
\hline $\bar{z}$ & $\bar{z}$ & $\bar{z}$ & $\bar{z}$ & & $=$ & $\bar{z}$ & $\bar{z}$ & $\bar{j}$ & - & $\overline{-}$ & - & - & 3 & - & - & - & 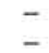 \\
\hline$\overline{-}$ & $\bar{z}$ & $\overline{-}$ & $\overline{-}$ & $N$ & $\overline{-}$ & $\overline{-}$ & $\overline{-}$ & 1 & $\overline{-}$ & $\overline{-}$ & 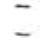 & $\bar{z}$ & $\bar{z}$ & $\bar{z}$ & $\overline{1}$ & $\bar{z}$ & 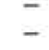 \\
\hline - & 1 & 1 & 1 & & 1 & 1 & 6 & 4 & 2 & - & - & - & - & 2 & - & - & \\
\hline - & - & - & - & & - & - & 2 & 2 & - & 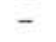 & - & & - & - & - & - & \\
\hline- & $\bar{z}$ & $\overline{1}$ & $\overline{1}$ & & $=$ & $\bar{j}$ & $\overline{1}$ & $\overline{3}$ & $\overline{3}$ & $\bar{z}$ & $\bar{z}$ & $\bar{z}$ & - & $\overline{3}$ & & - & \\
\hline$\overline{3}$ & $\overline{11}$ & 3 & $\begin{array}{c}1 \\
11\end{array}$ & & $\overline{6}$ & $\begin{array}{r}17 \\
17\end{array}$ & $\begin{array}{r}1 \\
15\end{array}$ & $\begin{array}{r}3 \\
17\end{array}$ & $\begin{array}{r}3 \\
18\end{array}$ & $\overline{13}$ & $\overline{27}$ & 4 & $\begin{array}{l}1 \\
22\end{array}$ & -3 & $\begin{array}{l}1 \\
6\end{array}$ & $\overline{23}$ & . \\
\hline - & - & - & - & & - & - & 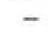 & 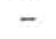 & 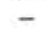 & - & & 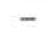 & - & - & - & 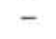 & \\
\hline$\overline{1}$ & $\overline{-}$ & 2 & 4 & & $\overline{-}$ & $\bar{v}$ & $\overline{6}$ & $\overline{-}$ & 3 & $\bar{z}$ & $\overline{1}$ & - & $\bar{z}$ & $\bar{z}$ & $\bar{z}$ & $\bar{z}$ & \\
\hline - & - & - & 3 & & 14 & 7 & 14 & 5 & 12 & 1 & 3 & - & - & - & - & 2 & \\
\hline & 2 & 1 & 1 & & - & 2 & 0 & 5 & & & & & & 1 & 2 & 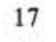 & \\
\hline- & $\bar{z}$ & $\bar{z}$ & - & & - & - & - & - & 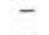 & - & 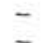 & 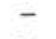 & - & - & - & - & \\
\hline$z$ & $\bar{z}$ & - & 1 & & $\overline{8}$ & & $\overline{1}$ & $\overline{8}$ & $\overline{4}$ & 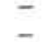 & - & - & & $\bar{z}$ & $1^{-}$ & $\bar{z}$ & \\
\hline 3 & 1 & 5 & 5 & & 10 & 6 & 23 & 40 & 16 & 25 & 23 & 5 & & 0 & 10 & 4 & \\
\hline - & $=$ & - & - & & - & - & - & - & - & - & - & & 1 & 1 & 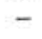 & - & \\
\hline$\overline{1}$ & $\bar{z}$ & - & I & & - & - & 1 & $\bar{z}$ & $\overline{1}$ & I & $\overline{2}$ & 1 & $\overline{4}$ & - & & - & \\
\hline & - & - & - & & - & - & - & 2 & - & & - & 1 & - & 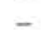 & & - & \\
\hline - & - & - & - & & - & - & - & - & - & - & - & & - & - & - & - & \\
\hline$\overline{-}$ & - & - & - & & - & - & 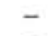 & - & - & - & - & - & - & - & & - & \\
\hline 2 & - & - & - & & - & - & - & - & - & 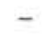 & 1 & - & - & - & - & - & \\
\hline 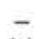 & - & - & - & & - & - & - & - & - & - & - & 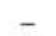 & - & - & - & - & \\
\hline 14 & 1 & 1 & 3 & & 6 & 2 & 11 & 2 & 4 & 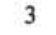 & 0 & 2 & 10 & 4 & 1 & 10 & \\
\hline _ & - & - & 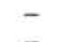 & & - & - & 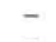 & - & - & - & - & $\overline{7}$ & - & 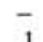 & - & 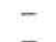 & \\
\hline- & - & - & - & & - & - & - & - & - & - & - & 1 & - & 1 & - & - & \\
\hline - & - & - & - & & - & - & - & - & - & - & - & - & - & - & - & - & \\
\hline- & - & 1 & - & & - & & & & & & & & & & & & \\
\hline- & - & - & - & & . & - & - & . & & . & & 1 & & - & & - & \\
\hline- & - & - & - & & & & - & - & & - & & 1 & & - & & - & \\
\hline- & - & - & - & & & - & - & - & & - & & - & & - & & - & \\
\hline 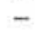 & - & - & - & & & - & - & - & & - & - & - & & & & - & \\
\hline - & & - & - & & . & & - & - & & - & . & - & & & & 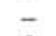 & \\
\hline 5 & - & - & 1 & & - & 1 & - & - & 1 & 3 & 2 & 4 & 2 & - & - & 2 & - \\
\hline - & - & - & - & & . & & - & - & - & & & & - & & & & \\
\hline 66 & 25 & 6 & 11 & & 10 & 1 & 2 & 4 & 17 & 12 & 20 & 3 & 6 & 4 & 4 & 18 & 1 \\
\hline & - & & & & & & & 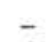 & & & & 2 & & - & - & & \\
\hline 93 & 47 & 2 & 1 & & & 2 & 1 & & 1 & & & & & . & & 21 & 3 \\
\hline & 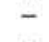 & 7 & - & & - & 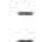 & - & - & 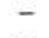 & . & 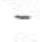 & - & - & - & - & - & 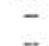 \\
\hline 7 & - & . & - & & & & 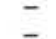 & $\overline{-}$ & $\bar{z}$ & & $\overline{1}$ & ? & & $=$ & & - & \\
\hline & 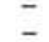 & 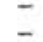 & - & & & & 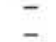 & $\bar{z}$ & $\overline{-}$ & 1 & 1 & 2 & 1 & & & $\bar{z}$ & \\
\hline & - & - & - & & & & - & - & & & & & - & & & - & \\
\hline & 1 & - & & & & & - & 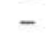 & - & & & & - & & & & \\
\hline & - & & & & & & - & - & & & & & & & & & \\
\hline & - & & - & & & & - & - & - & - & & & - & & & & \\
\hline & - & . & - & & & & & - & & - & & & - & & & & \\
\hline & - & - & - & & & & & - & & - & & & - & & & - & \\
\hline & - & - & & & & & & - & & - & & & - & & & & \\
\hline & - & - & & & & & - & - & & - & & & - & & & - & \\
\hline & - & - & - & & & & - & - & & - & & - & - & - & & 1 & \\
\hline & - & - & - & & & & & & & - & & & & 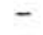 & & & \\
\hline- & - & - & - & & & & & - & & - & & & & & & - & \\
\hline- & - & - & - & & & & & - & & & & - & & & & - & \\
\hline 6 & - & 2 & 2 & & & & 2 & 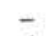 & & & & 4 & & & & 14 & \\
\hline & & - & - & & & & & & & - & & - & & - & - & - & \\
\hline & - & - & - & & & & & - & & - & & & & - & & - & \\
\hline & - & . & - & & & & & & & & & & & - & & 1 & \\
\hline & - & - & - & & & & & & & & & & & & & - & \\
\hline & - & - & - & & & - & - & - & & - & - & - & & - & & - & \\
\hline
\end{tabular}


TABLE 2 - Continued

\begin{tabular}{|c|c|c|c|c|c|c|c|c|c|c|c|c|c|c|c|c|c|c|c|c|}
\hline $380 \mathrm{~A}$ & 1 & 2 & 3 & 4 & 5 & 6 & 7 & 8 & 9 & 10 & 11 & 12 & 13 & 14 & 15 & 16 & 17 & 18 & 19 & 20 \\
\hline Fungal spores & _- & _- & 1 & - & 4 & - & - & - & - & 2 & 6 & - & - & 2 & - & - & - & - & 1 & - \\
\hline Dinoflagellates & & & & & & & & & & & & & & & & & & & & \\
\hline Undifferentiated & 1 & - & - & 6 & 1 & - & 1 & - & 9 & - & - & - & 1 & - & - & 3 & - & - & - & - \\
\hline Dino-19\&20 & 4 & - & 4 & 1 & - & - & 2 & - & 21 & - & - & 25 & 3 & 49 & 46 & 44 & 36 & 500 & 20 & 75 \\
\hline Botryococcus & - & - & - & - & - & - & - & - & - & - & - & - & - & - & 1 & - & - & - & - & - \\
\hline Pediastrum & 1 & - & - & 2 & - & 1 & - & - & - & 1 & - & - & 1 & - & - & - & - & - & - & - \\
\hline Acritarchs & & & & & & & & & & & & & & & & & & & & \\
\hline Undiferentiated & 3 & 10 & 5 & 9 & 6 & 1 & 2 & - & 17 & 2 & 7 & 6 & 13 & 12 & 10 & 6 & 1 & 1 & - & - \\
\hline Ac-1 & - & - & - & - & - & - & - & - & - & - & - & - & - & - & - & - & - & - & - & - \\
\hline Ac-8 & - & - & - & - & - & - & - & - & 1 & - & - & - & - & - & - & 1 & - & - & - & - \\
\hline Cymatiosphaera & - & - & - & - & - & - & - & - & - & - & - & - & - & - & - & - & - & - & - & - \\
\hline Tasmanites & - & - & _- & - & - & - & - & 1 & - & 3 & - & 1 & - & - & - & - & _- & - & - & - \\
\hline "Circuli" & - & - & - & - & - & - & - & - & - & - & - & - & - & - & - & - & - & - & - & - \\
\hline Trilete spores & & & & & & & & & & & & & & & & & & & & \\
\hline Undifferentiated & 2 & 3 & 1 & 2 & 6 & 5 & 1 & 2 & - & 6 & 3 & - & - & 1 & - & 1 & 6 & 1 & - & 1 \\
\hline Sphagnum & - & - & - & 2 & - & 1 & - & 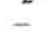 & 2 & - & - & 2 & 1 & 1 & - & 1 & 2 & - & - & - \\
\hline Klukisporites & - & - & - & - & - & - & - & - & - & - & - & - & - & - & - & - & - & - & - & - \\
\hline Monolete spores & 3 & 1 & 1 & 3 & 1 & 3 & - & - & 2 & 11 & 3 & 1 & 2 & 1 & - & - & - & 3 & 1 & - \\
\hline Undetermined pollen & 9 & 12 & 58 & 24 & 34 & 22 & 60 & - & 63 & 39 & 16 & 9 & 25 & 15 & 11 & 16 & 3 & 2 & 12 & 3 \\
\hline "Tetrad-1" & - & - & - & - & - & - & - & - & - & - & - & - & - & - & - & - & - & - & - & - \\
\hline Monocolpate & - & - & - & - & - & - & - & - & - & - & - & - & - & - & - & - & - & - & - & - \\
\hline Mesozoic-Paleogene triporates & - & 2 & - & 3 & 3 & 3 & - & - & - & 1 & 1 & 1 & 1 & - & - & - & 1 & - & - & - \\
\hline Conifers-Gymnosperms & & & & & & & & & & & & & & & & & & & & \\
\hline Bisaccate & 11 & 2 & 1 & 22 & 10 & - & 1 & 1 & 3 & 3 & 4 & 4 & 4 & 2 & - & - & 1 & 2 & - & - \\
\hline Inaperturate & - & 2 & 21 & 2 & 3 & 1 & 5 & 1 & - & - & 1 & - & - & - & 1 & - & - & - & - & - \\
\hline Cedrus & - & - & - & - & - & - & - & - & - & - & - & - & - & - & - & - & _- & - & - & - \\
\hline Keteleeria & - & - & - & - & - & - & - & - & - & - & - & - & - & - & - & - & - & - & - & - \\
\hline Picea-Abies & 7 & 1 & - & 3 & 2 & 3 & 1 & 2 & - & 5 & 11 & 4 & - & - & - & - & - & 2 & - & 1 \\
\hline Pinus & 33 & 6 & 12 & 30 & 30 & 46 & 16 & 3 & 1 & 36 & 29 & 39 & 33 & - & 2 & - & 1 & 1 & - & 9 \\
\hline Sciadopitys & - & - & - & - & - & - & - & - & - & - & - & - & - & - & - & - & - & - & - & - \\
\hline Sequoia & - & - & - & - & - & - & - & - & - & - & - & - & - & - & - & - & - & - & - & - \\
\hline Taxodiad & - & - & 5 & 3 & 1 & - & 1 & - & - & 1 & - & - & - & - & - & - & - & 1 & - & - \\
\hline Tsuga & - & - & - & - & - & - & - & - & - & - & - & - & - & - & - & - & - & - & - & - \\
\hline Vitreisporites & - & - & - & - & - & - & - & - & - & - & - & - & - & - & - & - & $\leftarrow$ & - & - & _- \\
\hline Corollina & - & - & - & - & - & - & - & - & - & - & - & - & - & - & - & - & _- & - & - & _- \\
\hline Ephedra & - & - & - & - & - & - & - & - & - & - & 1 & - & 1 & - & - & - & - & - & - & 1 \\
\hline Monocots & & & & & & & & & & & & & & & & & & & & \\
\hline Cyperaceae & - & - & - & - & _- & - & - & - & - & 2 & - & - & - & - & - & - & - & _- & - & _ \\
\hline Gramineae & 4 & 3 & 17 & 2 & 7 & 4 & 14 & - & - & 11 & 4 & 2 & 12 & - & 5 & - & 1 & _- & - & 6 \\
\hline Palmae & - & - & - & - & - & - & - & - & - & - & - & - & 1 & - & - & - & - & - & - & - \\
\hline Typha & - & - & - & - & - & - & - & - & - & - & - & - & - & - & - & - & - & - & - & - \\
\hline Dicots & & & & & & & & & & & & & & & & & & & & \\
\hline Alangium & - & _- & - & - & - & - & - & - & _- & - & - & _- & - & - & _- & - & - & - & - & _- \\
\hline Alnus & - & 1 & 13 & 2 & 2 & - & 4 & - & - & - & 2 & 1 & 1 & - & - & - & - & - & - & - \\
\hline Amaranthaceae & - & 2 & - & - & - & 1 & - & - & - & 1 & 3 & - & 2 & - & 7 & - & - & - & 3 & 2 \\
\hline Betula & - & - & - & - & - & 5 & 1 & - & 1 & - & - & - & 1 & - & - & 1 & - & - & - & - \\
\hline Carpinus - Ostrya & - & - & 15 & - & - & 1 & 11 & - & - & - & - & - & - & - & 1 & - & - & - & - & _- \\
\hline Carya & - & - & - & - & - & - & - & - & - & - & - & - & - & - & - & - & - & - & 1 & - \\
\hline Castanea & - & - & - & - & - & - & _- & - & - & - & - & - & - & - & - & - & - & - & - & _ \\
\hline Castanopsis & - & - & - & - & - & - & - & - & - & - & - & - & - & - & - & - & - & - & - & - \\
\hline Celtis & - & - & - & - & - & - & - & - & - & - & - & - & - & - & - & - & - & - & - & - \\
\hline Chenopodiaceae & 1 & 21 & 8 & 4 & 13 & 4 & 9 & 2 & 16 & 23 & 14 & 13 & 15 & 6 & 38 & 7 & 12 & 4 & 42 & 33 \\
\hline Compositae & & & & & & & & & & & & & & & & & & & & \\
\hline Undifferentiated & - & 1 & 2 & - & 3 & 2 & 2 & - & - & 2 & - & 2 & 8 & - & 3 & - & - & _- & - & 1 \\
\hline Artemisia & 3 & 30 & 26 & 12 & 27 & 19 & 28 & - & 18 & 23 & 14 & 5 & 7 & 3 & 16 & - & 2 & 3 & 22 & 26 \\
\hline Long-spined & - & - & - & - & - & - & - & - & 9 & - & - & - & - & - & - & - & - & - & - & - \\
\hline Fenestrate & - & - & - & - & - & - & - & - & - & - & - & - & - & - & - & - & - & - & - & - \\
\hline Corylus & 2 & - & 5 & 2 & - & 1 & 16 & - & - & 1 & 1 & - & 2 & - & - & - & - & - & - & - \\
\hline Engelhardia & - & - & - & - & - & - & - & - & - & - & - & - & - & - & - & - & - & - & - & - \\
\hline Ericaceae & - & - & 3 & - & 1 & - & - & - & - & - & - & - & - & - & - & - & - & - & - & - \\
\hline Fagus & - & - & 1 & - & - & - & - & - & - & 1 & - & - & - & - & - & - & - & - & - & - \\
\hline Fraxinus & - & _- & 9 & - & - & - & - & 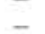 & - & - & 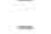 & - & - & - & 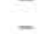 & 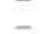 & - & - & - & - \\
\hline Ilex & - & - & - & - & - & - & - & - & - & - & - & - & - & - & - & - & - & _- & - & - \\
\hline Itea & - & - & - & - & - & - & - & - & - & - & - & - & - & - & - & - & - & - & - & - \\
\hline Labiatae & - & - & - & - & - & - & - & - & - & - & - & - & - & - & - & - & - & - & - & - \\
\hline Liquidambar & - & - & - & - & _- & _- & - & - & - & - & - & - & $\overline{-}$ & $=$ & - & - & - & $\overline{-}$ & $=$ & $\overline{-}$ \\
\hline Moraceae & - & - & - & - & - & - & - & - & - & - & - & - & - & - & - & - & - & - & - & - \\
\hline Myrica & - & - & 2 & - & - & - & - & - & - & - & _- & - & - & _- & _- & - & _- & _- & - & _- \\
\hline Nyssa & - & _- & - & - & - & - & _- & - & - & - & - & - & & - & _- & - & _- & - & - & - \\
\hline Parthenocissus & _- & _- & - & - & - & - & - & - & - & 1 & - & - & - & - & - & - & - & - & - & - \\
\hline Pterocarya & - & - & 1 & - & - & - & - & - & - & - & - & 2 & 1 & _- & - & - & _- & - & 6 & - \\
\hline Quercus & 2 & - & 13 & 2 & 1 & 1 & 32 & - & 5 & - & 6 & 3 & 3 & 5 & 1 & 1 & - & - & - & 1 \\
\hline Salix & - & - & - & - & - & - & - & - & - & - & - & - & - & - & - & - & - & - & - & - \\
\hline Tilia & - & - & - & - & - & - & - & - & - & 2 & 1 & - & 1 & - & - & - & - & - & - & - \\
\hline Ulmaceae & 1 & - & 5 & 2 & - & - & 4 & - & - & 2 & - & - & 2 & - & 1 & 1 & - & - & - & - \\
\hline Umbelliferae & - & - & - & - & - & 1 & - & - & - & - & - & - & - & - & - & - & - & - & - & - \\
\hline Zelkova & - & - & - & - & - & - & - & - & - & - & - & - & - & - & - & - & - & - & - & - \\
\hline
\end{tabular}


TABLE 2 - Continued

\begin{tabular}{|c|c|c|c|c|c|c|c|c|c|c|c|c|c|c|c|c|c|c|}
\hline 21 & 22 & 23 & 25 & 26 & 27 & 28 & 29 & 30 & 31 & 32 & 33 & 34 & 35 & 36 & 37 & 38 & 39 & 40 \\
\hline 1 & - & - & - & - & - & - & - & - & - & 1 & - & 2 & 1 & - & - & - & - & 1 \\
\hline 1 & - & - & - & - & 1 & - & - & - & - & - & 2 & - & 1 & 2 & 38 & 4 & 10 & 2 \\
\hline 60 & 25 & 250 & 2 & 100 & 75 & 90 & 80 & 3000 & 15 & 40 & - & - & 1 & - & 13 & - & 40 & - \\
\hline- & - & - & - & - & - & - & - & - & - & - & - & - & - & - & - & - & - & - \\
\hline- & - & - & - & - & - & - & - & - & - & - & - & - & - & - & - & - & - & - \\
\hline 6 & 2 & 2 & 3 & - & 3 & 5 & 2 & 2 & 15 & 2 & - & 21 & 4 & 1 & - & 1 & 55 & 150 \\
\hline- & - & - & - & - & - & - & - & - & - & - & - & - & - & - & - & - & - & - \\
\hline- & - & - & - & - & - & - & - & - & - & - & - & 80 & 20 & - & - & - & - & - \\
\hline - & - & - & - & - & - & - & - & - & - & - & - & - & - & - & - & - & - & - \\
\hline- & - & - & - & - & - & - & - & - & - & - & - & - & - & - & - & - & - & - \\
\hline- & - & - & - & - & - & - & - & - & - & - & - & - & - & - & - & - & - & - \\
\hline - & 1 & 1 & - & 1 & 1 & 1 & - & 1 & 1 & 1 & 1 & - & 2 & 1 & - & - & - & 1 \\
\hline- & - & - & - & - & 1 & - & - & - & - & - & - & - & - & - & 1 & - & - & - \\
\hline- & - & - & - & - & - & - & - & - & - & - & - & - & - & - & - & - & - & - \\
\hline- & 1 & 1 & - & - & - & 1 & - & - & 1 & 2 & - & - & 1 & 2 & - & - & - & 1 \\
\hline 13 & 17 & 23 & 7 & 6 & 2 & 10 & 10 & 10 & 9 & 4 & 11 & 17 & 36 & 14 & 1 & 3 & 13 & 18 \\
\hline- & - & - & - & - & - & - & - & - & - & - & - & - & - & - & - & - & - & - \\
\hline- & - & - & - & - & - & - & - & - & - & - & - & - & - & 1 & - & - & - & - \\
\hline - & - & - & - & - & - & - & 1 & - & - & - & - & - & - & - & - & - & - & - \\
\hline- & - & 1 & - & 2 & - & - & - & - & - & - & - & - & - & 1 & 1 & - & - & 1 \\
\hline- & - & 1 & - & - & - & - & - & - & - & - & - & 5 & - & 5 & - & - & 4 & - \\
\hline- & - & - & - & - & - & - & - & - & - & - & - & - & - & - & - & - & - & - \\
\hline- & - & - & - & - & - & - & - & - & - & - & - & - & - & - & - & - & - & - \\
\hline- & - & - & 2 & 10 & 6 & - & 5 & 1 & - & 2 & 11 & 3 & - & 2 & 4 & 1 & 29 & - \\
\hline 1 & 3 & 4 & 1 & 6 & 4 & 11 & 7 & 3 & 5 & 2 & 21 & 14 & 8 & 55 & 11 & - & 28 & 28 \\
\hline- & - & - & - & - & - & - & - & - & - & - & - & - & - & - & - & - & - & - \\
\hline- & - & - & - & - & - & - & - & - & - & - & - & - & - & - & - & - & - & - \\
\hline- & - & 1 & - & - & - & - & - & - & - & - & - & 3 & - & - & - & - & 1 & 1 \\
\hline - & - & - & - & - & - & - & - & - & - & 1 & - & 1 & - & 1 & - & - & 1 & 1 \\
\hline- & - & - & - & - & - & - & - & - & - & - & - & - & - & - & - & - & - & - \\
\hline- & - & - & - & - & - & - & - & - & - & - & - & - & - & - & - & - & - & - \\
\hline 1 & - & - & 1 & - & 1 & - & - & - & - & - & - & 1 & - & - & - & - & - & - \\
\hline - & - & - & - & - & - & - & - & - & - & - & - & - & - & - & - & - & - & - \\
\hline 2 & 1 & 2 & 2 & - & - & 1 & 3 & 6 & - & 2 & 1 & 52 & 28 & 18 & 2 & - & 5 & 8 \\
\hline- & - & - & - & - & - & - & - & - & - & - & - & - & - & - & - & - & - & - \\
\hline - & - & - & - & - & - & - & - & - & - & - & - & - & - & 1 & - & - & - & - \\
\hline- & - & - & - & - & - & - & - & - & - & - & - & - & - & - & - & - & - & - \\
\hline 1 & - & - & - & - & - & - & - & - & - & - & 3 & - & 2 & 6 & - & - & - & - \\
\hline 1 & 1 & - & 2 & 1 & - & 1 & - & 1 & 1 & - & - & 11 & 6 & - & - & - & - & 3 \\
\hline- & - & - & - & - & - & - & - & - & - & - & - & - & - & - & - & - & - & - \\
\hline- & - & - & - & - & - & - & - & - & - & - & - & 2 & 2 & - & - & - & - & - \\
\hline- & - & - & - & - & - & - & - & - & - & - & 1 & - & - & - & - & - & - & - \\
\hline- & - & - & - & - & - & - & - & - & - & - & - & - & - & - & - & - & - & - \\
\hline- & - & - & - & - & - & - & - & - & - & - & - & - & - & - & - & - & - & - \\
\hline- & - & - & - & - & - & - & - & - & - & - & - & - & - & - & - & - & - & - \\
\hline 27 & 57 & 39 & 25 & 27 & 13 & 26 & 23 & 30 & 15 & 5 & 25 & 76 & 62 & 15 & - & - & 7 & 27 \\
\hline 1 & 2 & 5 & 1 & 2 & 1 & - & 4 & - & - & 2 & 4 & 2 & 3 & 1 & - & - & - & 2 \\
\hline 11 & 10 & 15 & 40 & 3 & 3 & 17 & 9 & 17 & 14 & 2 & 5 & 23 & 34 & 5 & - & 1 & - & 10 \\
\hline- & - & - & - & - & - & - & - & 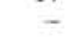 & - & - & - & 4 & - & - & - & - & - & - \\
\hline- & - & - & - & - & - & - & - & - & - & - & - & 2 & - & - & - & - & - & - \\
\hline- & - & 1 & - & 1 & - & - & - & - & - & 1 & 1 & 4 & 5 & 1 & - & - & - & - \\
\hline- & - & - & - & - & - & - & - & - & - & - & - & - & - & - & - & - & - & - \\
\hline- & - & - & - & - & 1 & - & - & - & - & - & - & 1 & - & 1 & - & - & - & - \\
\hline- & - & - & - & - & - & - & - & - & - & - & - & - & - & - & - & - & - & - \\
\hline- & - & - & - & - & - & - & - & - & - & - & - & - & - & - & - & - & - & - \\
\hline- & - & - & - & - & - & - & - & - & - & - & - & - & - & - & - & - & - & - \\
\hline- & - & - & - & - & - & - & - & - & - & - & - & - & - & - & - & - & - & - \\
\hline- & - & - & - & - & - & - & - & - & - & - & - & - & - & - & - & - & - & - \\
\hline- & - & - & - & - & - & - & - & - & - & - & - & - & - & - & - & - & - & - \\
\hline- & - & - & - & - & - & - & - & - & - & - & - & - & - & - & - & - & - & - \\
\hline- & - & - & - & - & - & - & - & - & - & - & - & - & - & - & - & - & - & - \\
\hline- & - & - & - & - & - & - & - & - & - & - & - & - & - & - & - & - & - & - \\
\hline- & - & 1 & - & - & - & - & - & - & - & - & - & - & - & - & - & - & - & - \\
\hline- & - & - & - & - & - & - & - & - & - & - & - & - & 1 & - & - & - & - & - \\
\hline 3 & 6 & - & 3 & - & 4 & - & 2 & - & - & - & 1 & 3 & 12 & 4 & 1 & - & 2 & 6 \\
\hline- & - & - & - & - & - & - & - & - & - & - & - & - & - & - & - & - & - & - \\
\hline- & - & - & - & - & - & - & - & 1 & - & - & - & - & - & - & - & - & - & - \\
\hline- & - & - & - & - & - & - & - & - & - & - & 1 & 1 & 1 & 9 & - & - & - & 3 \\
\hline- & - & - & - & - & - & - & - & - & - & - & - & 1 & - & - & - & - & - & - \\
\hline- & - & - & - & - & - & - & - & - & - & - & - & - & - & - & - & - & - & - \\
\hline
\end{tabular}


TABLE 2 - Continued

\begin{tabular}{|c|c|c|c|c|c|c|c|c|c|c|c|c|c|c|c|c|c|c|}
\hline & 41 & 42 & 43 & 44 & 45 & 46 & 47 & 48 & 49 & 50 & 51 & 52 & 53 & 54 & 55 & 56 & 57 & 58 \\
\hline Fungal spores & - & - & - & - & - & - & - & - & - & - & - & - & 1 & 2 & 1 & - & 1 & 1 \\
\hline \multicolumn{19}{|l|}{ Dinoflagellates } \\
\hline Undifferentiated & 6 & 17 & 14 & 9 & 16 & 2 & 10 & 35 & 1 & 40 & 40 & 46 & 42 & 70 & 22 & 66 & 2 & 3 \\
\hline Dino-19\&20 & - & 2 & 1 & 1 & 1 & - & - & 1 & - & - & 5 & - & 3 & 9 & - & - & - & - \\
\hline Botryococcus & - & 1 & 1 & - & - & - & - & - & - & 1 & - & - & - & - & - & - & - & - \\
\hline Pediastrum & - & - & - & - & - & - & - & - & - & - & - & - & - & - & - & - & - & 1 \\
\hline Acritarchs & & & & & & & & & & & & & & & & & & \\
\hline Undifferentiated & 100 & 104 & 42 & 33 & 20 & 7 & 7 & 19 & 77 & 39 & 19 & 33 & 25 & 103 & 240 & 118 & 708 & 32 \\
\hline Ac-1 & - & - & - & - & - & - & - & - & - & - & - & - & - & - & - & - & - & - \\
\hline Ac-8 & - & - & 69 & 45 & 15 & 28 & - & - & - & - & - & - & - & - & - & - & - & - \\
\hline Cymatiosphaera & - & - & - & - & - & - & - & - & - & - & - & - & - & - & - & - & - & - \\
\hline Tasmanites & - & - & - & - & - & - & - & - & - & - & - & - & - & - & - & - & - & - \\
\hline "Circuli" & - & - & - & - & - & - & - & - & - & - & - & - & - & - & - & - & - & - \\
\hline Trilete spores & & & & & & & & & & & & & & & & & & \\
\hline Undifferentiated & 1 & 1 & - & - & - & 3 & 1 & 4 & 2 & - & - & 1 & 3 & 2 & 1 & - & - & 1 \\
\hline Sphagnum & - & - & - & - & - & - & 1 & - & - & - & - & - & - & - & - & - & - & - \\
\hline Klukisporites & - & - & - & - & - & - & - & - & - & - & - & - & - & - & - & - & - & - \\
\hline Monolete spores & 2 & - & - & 1 & 1 & 1 & 4 & - & - & - & - & - & - & - & - & - & - & - \\
\hline Undetermined pollen & 10 & 28 & 46 & 25 & 26 & $16^{\circ}$ & 19 & 14 & 41 & 4 & 5 & 1 & 16 & 29 & 8 & 5 & 12 & 37 \\
\hline "Tetrad-1" & - & - & - & - & - & - & - & - & - & - & - & - & - & - & - & - & - & - \\
\hline Monocolpate & - & - & - & - & - & - & - & - & - & - & - & - & - & - & 1 & - & - & - \\
\hline Mesozoic-Paleogene triporates & - & 1 & - & - & - & - & - & - & 1 & - & - & - & 3 & - & - & - & - & - \\
\hline Conifers-Gymnosperms & & & & & & & & & & & & & & & & & & \\
\hline Bisaceate & - & - & - & - & - & - & - & - & - & - & - & - & 1 & - & - & - & - & 1 \\
\hline $\begin{array}{l}\text { Inaperturate } \\
\text { Cedrus }\end{array}$ & - & 1 & - & 2 & 1 & - & - & - & - & - & - & - & - & - & - & - & 2 & - \\
\hline Cedrus & - & - & - & - & - & - & - & - & - & - & - & - & - & - & - & - & - & - \\
\hline Keteleeria & - & - & - & - & - & - & - & - & - & - & - & - & - & - & - & - & - & - \\
\hline Picea-Abies & 18 & 9 & 3 & 1 & - & 6 & 10 & 2 & - & 1 & - & 2 & 2 & 3 & 1 & - & 2 & 3 \\
\hline Pinus & 21 & 58 & 74 & 24 & 34 & 48 & 21 & 36 & 5 & 18 & 51 & 43 & 24 & 28 & 39 & 20 & 26 & 6 \\
\hline Sciadopitys & - & - & - & - & - & - & - & - & - & - & - & - & - & - & - & - & - & - \\
\hline Sequoia & - & - & - & - & - & - & - & - & - & - & - & - & - & - & - & - & - & - \\
\hline Taxodiad & - & - & - & 1 & 3 & 5 & - & - & - & - & - & - & - & 1 & 1 & - & 1 & 1 \\
\hline Tsuga & 1 & - & 1 & 1 & 1 & 2 & 2 & - & - & - & 1 & - & 2 & 4 & 1 & - & - & 1 \\
\hline Vitreisporites & - & - & - & - & - & - & - & - & - & - & - & - & - & - & - & - & - & - \\
\hline Corollina & - & - & - & - & - & - & - & - & - & - & - & - & - & - & - & - & - & - \\
\hline Ephedra & - & 1 & - & - & - & - & - & - & 2 & - & - & - & 1 & 1 & - & - & 1 & - \\
\hline Monocots & & & & & & & & & & & & & & & & & & \\
\hline Cyperaceae & - & - & - & - & - & - & - & - & - & - & - & - & - & - & - & - & - & - \\
\hline Gramineae & 5 & 10 & 12 & 9 & 10 & 29 & 6 & 4 & 6 & - & - & 5 & - & 4 & 2 & 1 & 2 & 5 \\
\hline Palmae & - & - & - & - & - & - & - & - & - & - & - & - & - & - & - & - & - & - \\
\hline Typha & - & - & - & - & - & - & - & - & - & - & - & - & - & - & - & - & - & - \\
\hline Dicots & & & & & & & & & & & & & & & & & & \\
\hline Alangium & - & - & - & - & - & - & - & - & - & - & - & - & - & - & - & - & - & - \\
\hline Alnus & 1 & 2 & 2 & - & 4 & 2 & - & 1 & 1 & 2 & 2 & 1 & - & - & - & 1 & 2 & 8 \\
\hline Amaranthaceae & - & 3 & 2 & 7 & 3 & 3 & - & - & - & - & - & - & 1 & 2 & 1 & - & 1 & - \\
\hline Betula & - & - & - & - & 1 & - & - & - & - & - & - & - & - & - & - & - & - & _ \\
\hline Carpinus - Ostrya & - & 3 & 3 & 1 & 2 & 4 & - & 4 & - & 1 & - & 1 & - & - & - & 1 & - & 3 \\
\hline Carya & - & - & 1 & 2 & 1 & 2 & - & 4 & 3 & - & 1 & 2 & - & - & 1 & 1 & 1 & 1 \\
\hline Castanea & - & - & - & - & - & - & - & - & - & - & - & - & - & - & - & - & - & - \\
\hline Castanopsis & - & - & - & - & - & - & - & - & - & - & - & - & - & - & - & - & - & - \\
\hline Celtis & - & - & - & - & - & - & - & - & - & - & - & - & - & - & - & - & - & - \\
\hline Chenopodiaceae & 19 & 38 & 16 & 30 & 17 & 13 & 11 & 22 & 15 & 1 & 1 & 9 & 22 & 20 & - & 4 & 17 & 12 \\
\hline Compositae & & & & & & & & & & & & & & & & & & \\
\hline Undifferentiated & - & 3 & 1 & 5 & 2 & 1 & 1 & 4 & 1 & - & 2 & 4 & 2 & 1 & - & 1 & - & 1 \\
\hline Artemisia & 5 & 7 & 14 & 7 & 19 & 16 & 2 & 41 & 2 & 1 & 5 & 9 & 2 & 3 & - & 3 & 3 & 4 \\
\hline Long-spined & - & - & - & - & - & - & - & - & - & - & - & - & - & - & - & - & - & - \\
\hline Fenestrate & - & - & - & 1 & 1 & - & - & 1 & 1 & - & - & - & - & - & - & 1 & 1 & 1 \\
\hline Corylus & - & 1 & 2 & 1 & 3 & - & - & 1 & 2 & - & - & - & 1 & - & - & - & - & 1 \\
\hline Engelhardia & - & - & - & - & - & - & - & - & 1 & - & - & - & - & - & - & - & - & - \\
\hline Ericaceae & - & - & - & - & 2 & - & - & - & - & - & - & - & 1 & 1 & - & - & - & - \\
\hline Fagus & 1 & - & - & - & - & 2 & - & - & - & - & - & - & - & - & - & - & - & - \\
\hline Fraxinus & - & - & - & - & - & - & - & - & - & - & - & - & - & - & - & - & - & - \\
\hline nex & - & - & - & - & - & - & - & - & - & - & - & - & - & - & - & - & - & - \\
\hline Itea & - & - & _ & _- & - & - & - & - & - & - & _- & - & - & - & _- & - & - & - \\
\hline Labiatae & - & - & - & _- & - & - & - & - & - & - & - & - & - & - & _ & - & - & - \\
\hline Liquidambar & - & - & - & - & - & - & - & - & - & - & - & - & - & - & - & - & - & - \\
\hline Moraceae & - & - & - & - & - & - & - & - & - & - & - & - & - & - & - & - & - & - \\
\hline Myrica & - & - & - & - & - & - & - & - & - & - & - & - & 1 & - & - & - & - & 1 \\
\hline Nyssa & - & - & - & - & - & - & - & - & - & - & - & - & - & - & - & - & - & - \\
\hline Parthenocissus & - & - & - & - & - & - & - & - & - & - & - & - & - & - & - & - & - & - \\
\hline Pterocarya & 1 & - & - & - & - & - & - & 1 & - & - & - & - & - & - & - & - & 1 & - \\
\hline Quercus & 13 & 10 & 6 & 17 & 11 & 12 & 6 & 15 & 3 & 2 & 4 & 6 & 3 & 4 & 7 & 4 & 10 & 1 \\
\hline Salix & - & - & - & - & - & - & - & - & - & - & - & - & - & - & - & - & - & - \\
\hline Tilia & - & - & - & - & - & - & - & - & - & - & - & - & - & - & - & - & - & - \\
\hline Ulmaceae & 3 & 2 & - & 1 & - & 5 & 3 & 2 & 8 & 1 & - & 1 & 1 & 2 & - & 2 & 16 & 15 \\
\hline Umbelliferae & - & - & - & - & - & - & - & - & - & - & - & 1 & - & - & - & - & - & - \\
\hline Zelkova & - & - & - & - & - & - & - & - & - & - & - & - & - & - & - & - & - & - \\
\hline
\end{tabular}


TABLE 2 - Continued

\begin{tabular}{|c|c|c|c|c|c|c|c|c|c|c|c|c|c|c|c|c|c|c|c|c|c|}
\hline 59 & 60 & 61 & 62 & 63 & 64 & 65 & 66 & 67 & 69 & 70 & 71 & 72 & 73 & 74 & 75 & 76 & 77 & 78 & 79 & $\begin{array}{l}\mathrm{a} \\
80\end{array}$ & $\begin{array}{r}5 \\
80\end{array}$ \\
\hline- & 3 & - & 22 & - & 1 & 2 & - & 4 & 2 & 2 & 1 & - & 3 & 100 & 8 & 1 & 4 & 1 & 9 & - & - \\
\hline- & - & - & - & - & - & - & - & - & 1 & - & 1 & 1 & - & - & - & 1 & - & - & - & - & 1 \\
\hline 1 & $\bar{z}$ & $=$ & $\bar{z}$ & $=$ & $=$ & $\bar{z}$ & $\bar{z}$ & $\bar{z}$ & $\overline{-}$ & $\bar{z}$ & $\bar{z}$ & $\bar{z}$ & $\overline{-}$ & $\overline{-}$ & $\bar{z}$ & $\bar{z}$ & $\bar{z}$ & $\bar{z}$ & $\bar{z}$ & $\overline{-}$ & $\bar{z}$ \\
\hline$\overline{-}$ & $\overline{-}$ & $\overline{-}$ & $\overline{-}$ & $\bar{z}$ & $\bar{z}$ & $\overline{-}$ & $\overline{-}$ & $\bar{z}$ & $\overline{-}$ & $\bar{z}$ & $\overline{-}$ & $\overline{-}$ & $\bar{z}$ & $\overline{-}$ & $\bar{z}$ & $\bar{z}$ & $\overline{-}$ & $\overline{-}$ & $\overline{-}$ & $\overline{-}$ & $\overline{-}$ \\
\hline- & 84 & 21 & 15 & 2 & - & 1 & 1 & 8 & 168 & 23 & $\overline{9}$ & - & 3 & 24 & 10 & 21 & 19 & 16 & 16 & 4 & 8 \\
\hline $\bar{z}$ & $\bar{z}$ & $\bar{z}$ & $\overline{15}$ & $\bar{z}$ & $z$ & $\bar{z}$ & $\overline{-}$ & $\bar{z}$ & $\bar{z}$ & $\bar{z}$ & 57 & $\bar{z}$ & $\bar{z}$ & $\overline{-}$ & $\bar{z}$ & $\bar{z}$ & $=$ & $\bar{z}$ & - & $\overline{-}$ & $\bar{z}$ \\
\hline $\bar{z}$ & $\bar{z}$ & $\bar{z}$ & - & $\bar{z}$ & $\bar{z}$ & $\bar{z}$ & $\overline{-}$ & $\bar{z}$ & $\overline{-}$ & $\bar{z}$ & $\bar{z}$ & $\bar{z}$ & $\bar{z}$ & $\overline{-}$ & $\overline{-}$ & $\overline{-}$ & $\bar{z}$ & $\overline{-}$ & $\overline{-}$ & $\overline{-}$ & $\overline{-}$ \\
\hline - & - & - & - & - & - & - & 1 & - & - & - & - & - & - & - & - & & - & - & - & - & - \\
\hline- & - & - & - & - & - & - & - & - & - & - & - & - & - & - & - & -- & - & - & - & - & - \\
\hline 2 & - & - & - & - & 1 & 1 & - & 1 & 1 & 2 & 3 & $-^{*}$ & 4 & 1 & 3 & 1 & 2 & 2 & 2 & 2 & 1 \\
\hline $\bar{z}$ & $\bar{z}$ & $\bar{z}$ & $\overline{-}$ & $\bar{z}$ & $z$ & $\bar{z}$ & $\overline{-}$ & $z$ & 1 & $\bar{z}$ & $\bar{z}$ & $\bar{z}$ & $\bar{z}$ & $\overline{-}$ & $\bar{z}$ & $\bar{z}$ & $\overline{-}$ & $\bar{z}$ & $\overline{-}$ & - & - \\
\hline- & $=$ & $\overline{-}$ & $=$ & $\overline{-}$ & $\overrightarrow{1}$ & $=$ & $\overline{-}$ & $\overline{1}$ & 3 & $\overline{1}$ & $\overline{4}$ & $\overline{-}$ & $\overline{2}$ & 4 & $\overline{1}$ & $\overline{3}$ & $\overline{7}$ & $\overline{2}$ & $\bar{z}$ & $\overline{2}$ & $\overline{3}$ \\
\hline- & 16 & 41 & 107 & 19 & - & 28 & 72 & 48 & 110 & 49 & 74 & - & 24 & 67 & 18 & 68 & 33 & 30 & 33 & 10 & 12 \\
\hline- & - & - & - & - & - & - & - & 2 & 1 & 9 & 9 & - & 5 & 5 & $\overline{-}$ & 4 & 2 & 1 & 1 & 1 & 3 \\
\hline$\overline{-}$ & $\bar{z}$ & $\bar{z}$ & $\bar{z}$ & $\bar{z}$ & $\overline{1}$ & $\bar{z}$ & $\overline{-}$ & - & 3 & 2 & 15 & - & 8 & 12 & 2 & 11 & 4 & $\begin{array}{l}7 \\
1\end{array}$ & $\begin{array}{l}3 \\
1\end{array}$ & $\frac{2}{2}$ & $\begin{array}{l}4 \\
2\end{array}$ \\
\hline & & & & & - & & & - & - & 1 & - & - & - & 2 & 1 & 2 & 1 & & & & 2 \\
\hline$\overline{-}$ & $\bar{z}$ & $\bar{z}$ & $\begin{array}{r}26 \\
2\end{array}$ & $\bar{z}$ & $\overline{-}$ & $\bar{z}$ & $\overline{1}$ & $\overline{2}$ & - & - & - & $=$ & - & $-{ }_{3}$ & $\overline{1}$ & $\overline{1}$ & - & - & - & 2 & $\overline{3}$ \\
\hline$\overline{-}$ & $\overline{-}$ & - & - & - & - & - & -1 & 2 & $\bar{z}$ & $\bar{z}$ & $\bar{z}$ & $\bar{z}$ & $\overline{-}$ & ${ }_{-}^{3}$ & 1 & 1 & $\bar{z}$ & $\overline{-}$ & $\bar{z}$ & 1 & $\begin{array}{r}3 \\
-\end{array}$ \\
\hline- & - & - & - & - & 2 & - & - & 1 & - & - & - & - & - & - & - & - & - & - & - & - & $\overline{-}$ \\
\hline- & 9 & 11 & 4 & 13 & 23 & 16 & 26 & 12 & - & 4 & - & - & 9 & 3 & 3 & - & - & - & - & 7 & - \\
\hline- & 15 & 34 & 18 & 37 & 20 & 45 & 5 & 31 & 8 & 30 & 47 & 1 & 14 & 42 & 10 & 16 & 11 & 16 & 33 & 29 & 29 \\
\hline - & $\overline{-}$ & $\overline{-}$ & I & $\bar{z}$ & 1 & $=$ & 1 & $\overline{-}$ & $\overline{-}$ & $=$ & - & $\overline{-}$ & $\bar{z}$ & $\overline{-}$ & - & - & - & - & - & 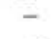 & $\overline{1}$ \\
\hline $\bar{z}$ & $\overline{1}$ & $\bar{z}$ & $\overline{-}$ & $\overline{1}$ & $\bar{z}$ & $\bar{z}$ & z & $\overline{1}$ & $\bar{z}$ & $\bar{z}$ & $\bar{z}$ & $\bar{z}$ & $\bar{z}$ & $\overline{-}$ & $\overline{-}$ & $\begin{array}{l}4 \\
1\end{array}$ & $\bar{z}$ & $=$ & $\bar{z}$ & $\overline{-}$ & 1 \\
\hline- & 1 & 2 & - & 1 & 1 & 1 & 2 & 1 & 1 & 2 & 3 & - & 3 & 3 & 1 & 1 & $\overline{1}$ & 2 & $\overline{1}$ & 1 & $\overline{1}$ \\
\hline- & - & $=$ & $\overline{-}$ & $\bar{z}$ & $\bar{z}$ & $\bar{z}$ & - & $\bar{z}$ & - & $=$ & - & - & - & - & - & - & - & - & - & - & - \\
\hline$\overline{-}$ & $\overline{-}$ & $\bar{z}$ & $\bar{z}$ & $\bar{z}$ & $z$ & $\bar{z}$ & $z$ & $z$ & $\bar{z}$ & $=$ & $\bar{z}$ & $\bar{z}$ & $\bar{z}$ & $\overline{-}$ & $\overline{-}$ & - & $=$ & $\bar{z}$ & $\bar{z}$ & $\bar{z}$ & $\overline{-}$ \\
\hline- & - & - & - & - & - & -. & - & - & - & & _ & - & 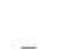 & 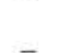 & & & & & & & - \\
\hline- & 3 & - & - & - & 1 & - & 1 & 10 & 1 & 4 & 1 & - & 4 & $\overline{10}$ & - & $\overline{1}$ & $\overline{2}$ & $\overline{2}$ & - & $\overline{1}$ & - \\
\hline- & - & - & - & - & - & - & - & - & - & - & - & - & - & - & 1 & 4 & 1 & 1 & - & $\begin{array}{l}1 \\
2\end{array}$ & $\overline{2}$ \\
\hline- & - & - & - & - & - & - & - & - & - & - & - & - & - & - & - & - & - & - & - & - & 1 \\
\hline- & - & - & - & - & - & - & - & - & - & - & - & - & - & - & 1 & - & - & - & - & - & - \\
\hline- & - & 1 & - & 1 & 3 & 1 & 1 & 2 & - & - & 2 & - & 1 & 3 & 5 & - & 1 & - & - & 1 & 2 \\
\hline- & - & - & - & 2 & - & - & $\bar{z}$ & $\bar{z}$ & $\bar{z}$ & 1 & - & - & - & 1 & $\overline{-}$ & $\overline{-}$ & $\bar{z}$ & $\bar{z}$ & $\overline{-}$ & $\overline{1}$ & $\overline{1}$ \\
\hline $\bar{z}$ & $\bar{z}$ & $\overline{1}$ & ${ }_{1}$ & $\overline{1}$ & $\overline{1}$ & $\overline{1}$ & $\overline{3}$ & $\overline{2}$ & $\bar{\tau}_{1}$ & $\overline{1}$ & $\bar{z}$ & $z$ & $\overline{1}$ & $\overline{-}$ & $\overline{1}$ & $\bar{z}$ & $\bar{z}$ & $\overline{-}$ & $\overline{-}$ & 1 & - \\
\hline- & 2 & - & - & 1 & 2 & - & - & - & - & - & 1 & - & 2 & 3 & 4 & - & - & 1 & - & 1 & 1 \\
\hline & - & - & - & - & & & - & & & & - & & 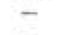 & - & 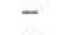 & - & - & 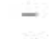 & & - & - \\
\hline$\overline{-}$ & $\bar{z}$ & $\bar{z}$ & $\bar{z}$ & $\bar{z}$ & $\bar{z}$ & $\bar{z}$ & $\overline{-}$ & $\overline{-}$ & - & $\overline{-}$ & $\overline{-}$ & $\bar{z}$ & $\bar{z}$ & $\overline{-}$ & $\overline{-}$ & - & $\bar{z}$ & $\bar{z}$ & $\bar{z}$ & $\bar{z}$ & $\bar{z}$ \\
\hline- & 3 & 12 & 20 & 3 & 2 & 1 & 11 & 11 & 1 & 3 & - & - & 1 & 4 & 4 & 3 & 1 & - & - & - & - \\
\hline- & 1 & - & - & 1 & - & 2 & 5 & 1 & 2 & 3 & - & 1 & 1 & - & - & - & - & 1 & - & - & - \\
\hline - & & & & - & 1 & - & & & 1 & 1 & 1 & - & & . & 1 & - & 1 & 1 & 1 & - & - \\
\hline- & - & - & - & - & - & - & - & - & $\bar{z}$ & - & - & - & $z$ & $\bar{z}$ & $\overline{1}$ & $\overline{-}$ & $z$ & $\bar{z}$ & _ & $\bar{z}$ & - \\
\hline- & $\overline{1}$ & $\overline{1}$ & $\bar{z}$ & $\bar{z}$ & $z$ & $\bar{z}$ & $\overline{1}$ & $\overline{3}$ & 8 & 7 & $\overline{8}$ & 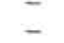 & $\overline{1}$ & 4 & $\begin{array}{l}1 \\
5\end{array}$ & $\overline{4}$ & 2 & 7 & $\overline{2}$ & $\overline{3}$ & $\overline{-}$ \\
\hline 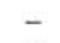 & - & - & 1 & - & - & - & - & 1 & - & - & $\begin{array}{l}0 \\
3\end{array}$ & - & - & - & - & 1 & - & 1 & - & 1 & - \\
\hline- & - & - & - & - & - & - & - & - & - & - & - & - & - & - & - & - & - & - & - & - & - \\
\hline- & - & - & - & - & - & - & - & - & 1 & - & - & - & - & 1 & - & - & - & - & - & - & - \\
\hline - & - & - & - & - & - & - & 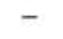 & - & - & - & - & & - & - & - & - & - & - & 1 & - & $?$ \\
\hline - & - & - & - & - & - & 1 & & - & - & - & - & & & & - & 1 & - & $\overline{1}$ & 1 & - & 2 \\
\hline- & - & - & - & - & - & - & - & 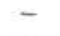 & - & - & - & $\overline{-}$ & - & & 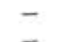 & $\sigma_{-}$ & - & 1 & - & - & - \\
\hline- & - & - & - & - & - & - & $\overline{-}$ & z & 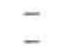 & $\bar{z}$ & $\overline{-}$ & $\overline{-}$ & $\bar{z}$ & - & $\overline{-}$ & - & $=$ & $\bar{z}$ & - & $\overline{1}$ & 1 \\
\hline- & $\bar{z}$ & $\bar{z}$ & $\bar{z}$ & $\overline{-}$ & $\bar{z}$ & $\bar{z}$ & $\bar{z}$ & - & $\bar{z}$ & $\bar{z}$ & $\overline{-}$ & $\overline{-}$ & $\bar{z}$ & 1 & $\overline{-}$ & $\bar{z}$ & 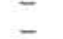 & $\bar{z}$ & $\bar{z}$ & 1 & 1 \\
\hline $\bar{z}$ & $\overline{-}$ & $\bar{z}$ & 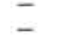 & $\overline{-}$ & - & $\overline{-}$ & $\bar{z}$ & $\overline{-}$ & - & - & - & 1 & - & - & $\overline{4}$ & $\overline{-}$ & - & - & - & 1 & $\bar{z}$ \\
\hline - & - & - & - & - & - & - & - & - & - & - & - & - & - & - & - & - & - & - & - & - & - \\
\hline 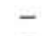 & - & - & - & - & - & - & - & - & 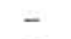 & - & - & - & - & 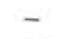 & - & - & - & 1 & - & - & - \\
\hline & 3 & - & - & - & - & 2 & - & 3 & - & - & - & - & - & - & - & - & - & - & - & 1 & 1 \\
\hline & 7 & 4 & 1 & 5 & 4 & 2 & 4 & 15 & 5 & 12 & 5 & - & 3 & 5 & 4 & 1 & 3 & 2 & 1 & 5 & 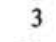 \\
\hline & - & - & - & - & - & - & - & - & - & - & - & - & - & 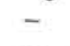 & 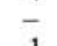 & - & - & $\overline{-}$ & 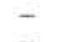 & - & - \\
\hline$\overline{1}$ & $\overline{5}$ & $\overline{11}$ & - & 1 & $\overline{4}$ & $\overline{4}$ & $\overline{10}$ & $\overline{26}$ & $\overline{-}_{4}$ & $\overline{3}$ & $\overline{1}$ & $\bar{i}$ & $\overline{2}$ & $-{ }_{4}$ & 1 & $\overline{4}$ & $\overline{-}$ & 1 & $\overline{2}$ & $\overline{1}$ & 2 \\
\hline 1 & 3 & 11 & ${ }_{-}^{2}$ & $\frac{4}{-}$ & $\frac{4}{-}$ & -4 & 10 & - & $\begin{array}{l}4 \\
1\end{array}$ & - & 1 & $\frac{1}{-}$ & 2 & ${ }^{4}$ & - & - & $\bar{z}$ & $\bar{z}$ & - & - & - \\
\hline- & - & - & - & - & - & - & - & - & - & - & - & - & - & - & - & - & - & - & - & - & - \\
\hline
\end{tabular}


TABLE 3

Palynological Analyses, Site 381

\begin{tabular}{|c|c|c|c|c|c|c|c|c|c|c|c|c|c|c|c|c|c|c|c|c|}
\hline & 1 & 2 & 3 & 4 & 5 & 6 & 7 & 8 & 9 & 10 & 11 & 12 & 13 & 14 & 15 & 16 & 17 & 18 & 19 & 20 \\
\hline Fungal spores & - & 7 & 10 & 1 & 1 & 2 & 10 & 8 & 2 & 13 & 13 & 2 & - & 14 & 1 & 4 & - & 9 & - & 9 \\
\hline Dinoflagellates & & & & & & & & & & & & & & & & & & & & \\
\hline Undifferentiated & - & 3 & 2 & 1 & - & - & 6 & - & 4 & 1 & - & 3 & - & 17 & 2 & 7 & 19 & 18 & 1 & - \\
\hline Dino-11 & - & - & - & - & - & - & - & - & - & - & - & - & 5 & - & - & - & - & - & - & - \\
\hline Dino-14 & - & - & - & - & - & - & - & - & - & _- & - & - & - & 7 & - & - & - & - & - & - \\
\hline Dino-18 & - & - & - & - & - & - & - & - & 1 & - & - & - & - & - & - & 6 & - & - & - & - \\
\hline Dino-19\&20 & - & - & - & _- & - & - & 7 & 5 & 63 & 3 & 1 & 1 & 50 & 20 & 341 & 855 & 5 & 54 & 25 & 7 \\
\hline Dino-22 & - & - & - & - & _ & - & - & - & - & - & - & - & - & - & - & - & - & 8 & - & - \\
\hline Dino-24 & - & - & - & - & - & - & - & - & - & - & - & - & - & - & - & - & - & - & - & - \\
\hline Dino-24a & - & - & - & - & - & - & - & - & - & - & - & - & - & - & - & - & - & - & - & - \\
\hline Dino-24b & - & - & - & - & - & - & - & - & - & - & - & - & - & - & - & - & - & - & - & - \\
\hline Dino- 25 & - & - & - & - & - & - & - & - & - & - & - & - & - & - & - & - & - & - & - & - \\
\hline Dino-26 & - & - & - & _- & - & - & - & - & - & - & - & - & - & - & - & - & - & - & 2 & - \\
\hline Dino-28 & - & - & - & - & - & - & - & - & - & - & _ & _ & - & - & - & - & - & - & - & - \\
\hline Dino-29 & - & - & - & _- & - & - & - & - & - & - & _ & - & - & - & - & - & - & - & - & - \\
\hline Botryococcus & - & - & - & - & - & - & - & - & - & - & - & - & - & - & - & - & - & - & - & - \\
\hline Pediastrum & - & - & - & - & - & - & - & - & - & - & - & - & - & - & - & - & - & - & - & - \\
\hline Acritarchs & & & & & & & & & & & & & & & & & & & & \\
\hline Undifferentiated & 15 & 1 & 3 & 2 & 3 & - & - & 1 & 25 & 6 & - & 3 & 2 & 19 & 2 & - & 6 & 94 & 1 & 15 \\
\hline $\mathrm{Ac}-4$ & - & - & - & - & - & - & - & - & - & - & - & - & - & - & - & - & - & - & - & - \\
\hline Ac- 8 & - & - & - & - & - & - & - & - & - & - & - & - & _- & - & - & - & - & - & - & 131 \\
\hline$A c-10 b$ & - & - & - & - & - & - & - & - & - & - & - & - & - & - & - & - & - & - & - & - \\
\hline Ac- 11 & - & - & - & _- & - & _- & - & - & - & - & - & - & - & - & - & - & - & - & - & - \\
\hline $\mathrm{Ac}-12$ & - & - & - & - & - & - & - & - & - & - & - & - & - & - & - & - & - & - & - & - \\
\hline Cymatiosphaera & - & - & - & - & - & - & - & - & - & - & - & - & - & - & - & - & 1 & - & - & - \\
\hline Trilete spores & & & & & & & & & & & & & & & & & & & & \\
\hline Undifferentiated & - & - & - & - & 1 & - & - & - & - & 2 & 1 & - & 1 & 3 & - & - & 2 & - & - & 2 \\
\hline Sphagnum & - & - & - & - & - & - & 1 & - & 1 & - & - & - & - & - & - & - & $i$ & 1 & - & - \\
\hline Monolete spores & 5 & - & - & - & 1 & - & - & 1 & 6 & - & 1 & 2 & - & 3 & - & - & 1 & 1 & - & 1 \\
\hline Undetermined pollen & 14 & 47 & 100 & 48 & 10 & 51 & 117 & 112 & 25 & 66 & 58 & 40 & 1 & 51 & 30 & 15 & 11 & 30 & 2 & 16 \\
\hline "Tetrad-1" & - & - & - & - & - & - & - & - & - & - & - & - & - & - & - & - & - & - & - & - \\
\hline Monocolpate & - & - & - & - & 1 & - & - & - & - & - & - & - & - & - & 1 & - & 1 & - & - & - \\
\hline Mesozoic-Paleogene triporates & - & - & - & - & - & - & - & _- & - & 1 & - & 1 & - & - & - & - & - & 1 & - & - \\
\hline Pc3-10 & - & - & - & - & - & - & - & - & - & - & - & - & - & - & - & - & - & - & - & - \\
\hline Conifers-Gymnosperms & & & & & & & & & & & & & & & & & & & & \\
\hline Bisaccate & - & 1 & 1 & _- & - & 3 & 4 & 13 & 2 & 4 & 23 & 15 & - & 4 & 1 & _- & _- & 2 & - & - \\
\hline Inaperturate & 14 & - & 2 & 2 & 1 & - & - & 11 & 1 & 6 & - & 3 & - & 9 & - & 1 & - & - & - & - \\
\hline Cedrus & - & 1 & 1 & 1 & - & 3 & - & - & - & 4 & 8 & 1 & - & - & - & - & - & - & - & - \\
\hline Keteleeria & - & - & - & - & - & - & - & - & - & - & - & - & - & - & - & - & - & - & - & - \\
\hline Picea-Abies & - & - & - & - & - & - & - & - & - & 1 & - & - & - & - & - & - & 4 & - & - & 1 \\
\hline Pinus & 5 & 5 & 7 & 3 & 17 & 3 & 3 & 4 & 10 & 20 & 11 & 9 & 6 & 3 & 2 & 11 & 14 & 1 & - & 8 \\
\hline Podocarpus & - & - & - & - & - & - & - & - & - & - & - & - & - & - & - & - & - & - & - & - \\
\hline Sciadopitys & - & - & - & - & - & - & - & - & - & - & - & - & - & - & - & - & - & - & - & - \\
\hline Sequoia & - & - & - & - & - & - & - & - & - & - & - & - & - & - & - & - & 1 & - & - & - \\
\hline Taxodiad & 5 & - & - & - & - & - & - & - & - & - & - & - & - & - & - & - & - & - & - & - \\
\hline Tsuga & - & - & - & - & - & - & - & - & - & - & - & - & - & - & - & _ & 1 & - & - & - \\
\hline Vitreisporites & - & - & - & - & - & - & - & - & - & - & - & - & - & - & - & - & - & - & - & - \\
\hline Corollina & - & - & - & - & - & - & - & - & - & 1 & - & - & - & - & - & _ & - & - & - & - \\
\hline Ephedra & - & - & - & - & - & - & - & - & - & - & _- & 1 & - & - & - & _ & - & - & - & - \\
\hline Moncots & & & & & & & & & & & & & & & & & & & & \\
\hline Gramineae & 4 & 9 & 5 & 4 & - & 3 & 5 & 1 & - & 3 & 3 & 1 & - & 13 & - & - & - & 7 & - & 6 \\
\hline Typha & 2 & - & 1 & - & - & - & - & - & - & 1 & - & - & - & - & - & - & - & - & - & - \\
\hline Dicots & & & & & & & & & & & & & & & & & & & & \\
\hline Alnus & 1 & 1 & - & - & - & - & - & - & - & 1 & 2 & - & - & 1 & - & - & 4 & 1 & - & - \\
\hline Amaranthaceae & - & 1 & 6 & 1 & - & - & 1 & 4 & - & 1 & 4 & 3 & - & 4 & - & 1 & - & 3 & - & 4 \\
\hline Betula & 1 & - & - & 1 & - & - & 1 & - & - & 1 & - & 1 & - & - & - & - & 2 & 1 & - & - \\
\hline Carpinus - Ostrya & 1 & - & - & - & - & - & - & - & 1 & - & - & 1 & - & - & - & - & - & - & - & - \\
\hline Carya & - & - & - & - & - & - & - & - & - & - & - & - & - & - & - & - & - & - & - & - \\
\hline Caryophyllaceae & - & - & - & 1 & - & - & - & - & - & - & - & - & - & - & - & - & - & - & - & - \\
\hline Chenopodiaceae & 30 & 25 & 41 & 38 & 8 & 15 & 16 & 32 & 3 & 3 & 16 & 14 & 3 & 34 & 12 & 8 & 3 & 36 & 4 & 24 \\
\hline Compositae & & & & & & & & & & & & & & & & & & & & \\
\hline Undifferentiated & 2 & - & - & - & - & - & - & - & 1 & - & - & - & - & - & - & _ & - & - & - & _- \\
\hline Artem & 30 & 90 & 76 & 110 & 39 & 31 & 17 & 39 & 6 & 12 & 31 & 15 & 1 & 2 & 26 & 4 & 1 & 8 & - & 9 \\
\hline Long-spined & - & 1 & 3 & 2 & - & 1 & - & 5 & - & - & 6 & 3 & - & 4 & - & 1 & - & 5 & - & - \\
\hline Fenestrate & - & - & 1 & - & - & - & - & 1 & - & - & - & - & - & 1 & - & 1 & - & - & - & - \\
\hline Corylus & 2 & - & - & - & 3 & - & - & - & - & - & - & - & - & - & - & - & 4 & - & - & - \\
\hline Engelhardia & - & - & - & - & - & - & - & - & - & - & - & 1 & - & - & - & - & - & - & - & - \\
\hline Fagus & - & 1 & - & - & - & - & - & - & - & - & - & - & - & - & - & - & - & - & - & - \\
\hline Ilex & - & - & - & - & - & - & - & - & - & - & - & - & - & - & - & - & - & - & - & - \\
\hline Myrica & - & - & - & - & - & - & - & - & - & - & - & - & - & - & - & - & 1 & - & - & - \\
\hline Pterocarya & - & - & - & - & 1 & - & - & - & - & - & - & - & - & - & - & - & 1 & - & - & - \\
\hline Quercus & 30 & - & 1 & - & 7 & - & - & - & - & - & - & - & 1 & 2 & 1 & 3 & 8 & - & - & - \\
\hline Schizandra & - & - & - & - & - & - & - & - & - & - & - & - & - & - & - & - & - & - & - & - \\
\hline Tilia & - & - & - & - & - & - & - & - & - & - & - & - & - & - & - & - & - & - & - & - \\
\hline Ulmaceae & - & - & 1 & - & - & - & - & - & - & - & 1 & - & - & 1 & - & - & 2 & - & - & - \\
\hline Umbelliferae & 1 & - & - & 1 & - & - & - & - & - & - & - & - & - & - & - & - & - & - & - & - \\
\hline
\end{tabular}


TABLE 3 - Continued

\begin{tabular}{|c|c|c|c|c|c|c|c|c|c|c|c|c|c|c|}
\hline 22 & 23 & 24 & 25 & 26 & 27 & 28 & 29 & 30 & 31 & 32 & 33 & 34 & 35 & 36 \\
\hline- & 9 & - & 10 & 2 & 6 & - & 12 & 1 & 3 & - & 20 & - & 3976 & - \\
\hline 2 & 36 & 6 & 12 & 8 & 51 & 17 & 44 & 3 & 24 & 56 & 21 & 14 & 31 & 150 \\
\hline $\bar{z}$ & $\bar{z}$ & $=$ & $\overline{-}$ & - & - & - & - & - & - & - & - & $=$ & $=$ & $\overline{-}$ \\
\hline 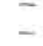 & 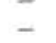 & $\overline{-}$ & 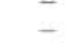 & $\overline{-}$ & $\overline{-}$ & $\overline{-}$ & $\overline{-}$ & $\overline{-}$ & $z$ & $\bar{z}$ & $\overline{-}$ & 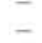 & $=$ & 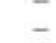 \\
\hline- & 8 & 6 & 1 & 2 & - & - & - & - & - & 56 & 2 & 1 & - & 90 \\
\hline- & & - & - & - & - & - & $\overline{0}$ & - & $\overline{0}$ & - & - & - & - & - \\
\hline & 000 & - & $-\overline{-}$ & - & - & - & 21 & - & 3 & - & - & - & - & - \\
\hline - & - & - & 1250 & - & $\overline{6}$ & - & - & - & - & - & - & - & - & - \\
\hline - & $=$ & - & - & - & 60 & - & - & - & - & - & - & - & - & - \\
\hline - & $=$ & $=$ & $\overline{-}$ & - & - & - & - & - & - & - & 18 & - & - & - \\
\hline - & & - & - & - & - & & - & - & 40 & - & 5 & - & - & - \\
\hline- & - & - & $\bar{z}$ & - & - & - & - & - & - & - & 22 & - & - & - \\
\hline- & & - & - & - & - & - & - & - & - & - & - & - & - & - \\
\hline - & - & 1 & - & - & - & 1 & - & - & - & - & - & - & - & - \\
\hline- & - & - & - & - & - & - & - & - & - & - & - & - & $=$ & - \\
\hline 6 & - & 8 & - & 15 & 10 & 10 & 64 & 15 & 9 & 140 & 13 & 54 & 33 & 800 \\
\hline- & - & - & - & - & - & - & - & - & - & - & - & - & 50 & - \\
\hline- & - & - & - & - & 48 & - & 6 & - & 14 & - & 6 & - & 1 & - \\
\hline - & - & - & - & - & - & - & - & - & - & - & - & - & - & - \\
\hline - & & - & - & - & - & - & - & - & - & - & - & - & 266 & - \\
\hline - & - & - & - & - & - & - & - & - & - & - & - & - & - & - \\
\hline- & - & - & - & - & - & - & - & - & - & - & - & 1 & - & 1 \\
\hline - & - & - & - & 1 & 1 & - & - & 1 & 1 & 4 & - & 1 & - & - \\
\hline- & - & - & - & - & - & - & - & - & 1 & - & - & - & - & - \\
\hline$\overline{-}$ & $\bar{c}$ & $\overline{-}$ & $\vec{u}$ & - & $\bar{z}$ & 2 & - & 1 & - & $\overline{-}$ & $\overline{-}$ & $\overline{-}$ & - & - \\
\hline 8 & 5 & 12 & 14 & 14 & 26 & 13 & 36 & 15 & 21 & 12 & 56 & 10 & 19 & 1 \\
\hline$\overline{2}$ & $\overline{-}$ & $\bar{I}$ & I & - & $\overline{1}$ & 1 & - & $\begin{array}{l}2 \\
1\end{array}$ & $\overline{-}$ & $\overline{1}$ & $\overline{-}$ & $\overline{-}$ & ${ }_{1}$ & $\overline{-}$ \\
\hline- & - & - & 2 & - & - & - & 3 & 1 & - & - & - & - & - & - \\
\hline- & - & - & - & - & - & - & - & - & - & - & - & - & - & - \\
\hline- & 1 & - & - & - & 2 & - & - & - & - & - & - & - & - & - \\
\hline 2 & - & - & - & 5 & 3 & 5 & - & - & 1 & - & - & 2 & - & - \\
\hline- & - & - & - & - & - & - & - & - & - & - & - & - & - & - \\
\hline $\overrightarrow{10}$ & - & $\overline{6}$ & $=$ & $\overline{7}$ & $\overline{1}$ & 7 & - & $\overline{1}$ & - & $\overline{1}$ & - & $\overline{6}$ & - & $\overline{2}$ \\
\hline $\begin{array}{r}10 \\
4\end{array}$ & $\overline{6}$ & $\begin{array}{l}6 \\
2\end{array}$ & $\overline{5}$ & 29 & $\begin{array}{r}1 \\
10\end{array}$ & $\begin{array}{r}7 \\
25\end{array}$ & $\overline{51}$ & $\begin{array}{r}1 \\
26\end{array}$ & $\overline{17}$ & $\begin{array}{r}1 \\
29\end{array}$ & 3 & $\begin{array}{r}6 \\
12\end{array}$ & 49 & 27 \\
\hline- & - & - & - & - & - & - & - & - & - & - & - & - & - & - \\
\hline - & - & - & - & - & - & - & - & - & - & - & - & - & - & - \\
\hline- & - & - & - & - & - & 1 & - & - & - & - & - & 1 & - & - \\
\hline- & - & - & - & 1 & - & 4 & - & - & - & 1 & - & - & - & - \\
\hline- & - & - & - & - & - & - & 1 & - & - & 2 & - & - & 1 & - \\
\hline - & - & - & - & - & & & - & - & - & 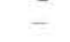 & - & - & - & - \\
\hline- & - & - & - & - & - & - & - & - & - & - & - & - & - & - \\
\hline - & - & - & - & - & - & - & - & - & - & - & - & - & - & - \\
\hline- & 1 & - & 15 & - & 8 & 4 & 4 & - & 10 & - & 4 & 1 & 2 & 1 \\
\hline- & - & - & - & - & 1 & - & - & - & - & - & - & - & - & - \\
\hline - & - & - & 1 & - & 3 & - & 1 & 1 & 1 & - & 1 & - & 5 & - \\
\hline - & - & 1 & 4 & - & 10 & - & 1 & - & 3 & - & - & - & - & - \\
\hline - & - & - & 1 & - & 1 & - & $\overline{-}$ & - & - & - & - & - & - & - \\
\hline - & - & - & 1 & - & - & - & 1 & - & 2 & - & - & - & 1 & 2 \\
\hline - & - & - & 2 & 2 & - & 1 & 1 & - & - & - & - & - & 3 & - \\
\hline$\overline{0}$ & $\bar{j}$ & $\overline{0}$ & $\bar{u}$ & & - & $\overline{-}$ & - & - & - & $\overline{8}$ & $\overrightarrow{35}$ & - & - & $\overline{1}$ \\
\hline 22 & 1 & 28 & 44 & 12 & 33 & 11 & 9 & 6 & 6 & 8 & 35 & - & 1 & 1 \\
\hline- & - & - & $=$ & 3 & - & 1 & - & 1 & - & 1 & - & 1 & 1 & - \\
\hline 2 & 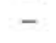 & 5 & 3 & 2 & 5 & 8 & 7 & - & 4 & 3 & 16 & - & - & - \\
\hline & - & - & 4 & - & 4 & - & 2 & - & - & - & 5 & - & - & 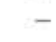 \\
\hline- & - & - & 2 & - & - & - & - & - & - & - & - & - & - & - \\
\hline 1 & - & - & - & 1 & - & 2 & - & 2 & - & - & - & - & 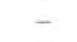 & - \\
\hline & - & - & - & - & - & - & - & - & - & - & - & - & - & - \\
\hline- & - & - & - & - & - & - & - & - & - & - & - & - & - & - \\
\hline - & - & - & - & - & - & - & - & 1 & - & - & - & - & - & - \\
\hline- & - & - & - & - & - & - & 1 & - & - & - & 1 & - & 3 & - \\
\hline - & 1 & - & - & - & 1 & 4 & - & - & - & - & 1 & - & 2 & - \\
\hline 12 & - & 5 & 6 & 9 & 6 & 13 & 16 & 2 & 15 & 4 & 9 & - & 32 & - \\
\hline & - & - & & 1 & -- & - & - & - & - & - & - & - & - & - \\
\hline- & - & - & - & - & - & - & - & - & - & - & - & - & - & - \\
\hline- & 1 & 1 & 1 & 1 & 1 & 4 & - & 1 & - & - & - & 2 & 10 & - \\
\hline - & - & - & - & - & i & - & - & - & - & - & - & - & - & - \\
\hline
\end{tabular}


TABLE 3 - Continued

\begin{tabular}{|c|c|c|c|c|c|c|c|c|c|c|c|c|c|c|}
\hline & 37 & 38 & 39 & 40 & 43 & 44 & 45 & 46 & 48 & 49 & 51 & 52 & 16 & 21 \\
\hline Fungal spores & 7 & - & 6 & - & 12 & & 12 & & 28 & 105 & 15 & 17 & 16 & 21 \\
\hline \multicolumn{15}{|l|}{ Dinoflagellates } \\
\hline Undifferentiated & 21 & - & 6 & 1 & - & & 10 & & 9 & 16 & 9 & - & 6 & 8 \\
\hline Dino-11 & - & - & - & - & - & & - & & - & - & - & - & - & - \\
\hline Dino-14 & - & - & - & - & - & & - & & - & - & - & - & - & - \\
\hline Dino-18 & - & - & - & - & - & & - & & - & - & - & - & - & - \\
\hline Dino-19\&20 & - & 7 & - & - & - & & - & & - & - & - & - & 2 & - \\
\hline Dino-22 & - & - & - & - & - & & - & & - & - & - & - & - & - \\
\hline Dino-24 & - & - & - & - & - & & - & & - & - & - & - & - & - \\
\hline Dino-24a & - & - & - & - & - & & - & & - & - & - & - & - & - \\
\hline Dino-24b & - & - & - & - & - & & - & & - & - & - & - & - & - \\
\hline Dino-25 & - & - & - & - & - & & - & & - & - & - & - & - & - \\
\hline Dino-26 & 1 & - & - & - & - & & - & & - & - & - & - & - & - \\
\hline Dino-28 & - & - & - & - & - & & - & & - & - & - & - & - & - \\
\hline Dino-29 & - & 3600 & - & - & - & & - & & - & - & - & - & - & - \\
\hline Botryococcus & - & - & - & - & 1 & & - & & - & - & - & - & - & - \\
\hline Pediastrum & - & - & - & 4 & - & & - & & 1 & - & - & - & - & - \\
\hline \multicolumn{15}{|l|}{ Acritarchs } \\
\hline Undifferentiated & 94 & 452 & 23 & 4 & 10 & & 14 & & 21 & 25 & 13 & 17 & 9 & 5 \\
\hline$A c-4$ & 46 & - & - & - & - & & - & & - & - & - & - & - & $\overline{-}$ \\
\hline Ac-8 & - & - & 5 & - & 3 & & 18 & & 9 & 15 & 5 & 1 & 2 & 12 \\
\hline$A c-10 b$ & 1300 & - & - & - & - & & - & & - & - & - & - & - & - \\
\hline Ac-11 & - & - & - & - & - & & - & & - & - & - & - & - & - \\
\hline Ac- 12 & - & - & - & - & - & & - & & - & 47 & - & - & 1 & - \\
\hline Cymatiosphaera & - & - & - & - & - & & - & & - & - & - & - & - & - \\
\hline Trilete spores & & & & & & B & & B & & & & & & \\
\hline Undifferentiated & - & - & - & 1 & 1 & A & - & A & 2 & - & 7 & 3 & 8 & 1 \\
\hline Sphagnum & - & - & - & - & - & $\mathbf{R}$ & - & $\mathbf{R}$ & - & - & - & - & - & - \\
\hline Monolete spores & - & - & 2 & - & 5 & R & - & $\mathbf{R}$ & 1 & 1 & 4 & 5 & 6 & 7 \\
\hline Undetermined pollen & 15 & 7 & 88 & 8 & 42 & $\mathrm{E}$ & 23 & $\mathrm{E}$ & 75 & 104 & 97 & 80 & 88 & 61 \\
\hline "Tetrad-1" & - & - & - & - & - & $\mathrm{N}$ & - & $\mathrm{N}$ & - & - & - & - & - & $\overline{0}$ \\
\hline Monocolpate & - & - & 4 & - & 1 & & - & & 6 & 7 & 4 & - & - & 2 \\
\hline Mesozoic-Paleogene triporates & - & - & - & - & 1 & & - & & - & 1 & - & - & - & 1 \\
\hline \multirow{2}{*}{\multicolumn{15}{|c|}{ Conifers-Gymnosperms }} \\
\hline & & & & & & & & & & & & & & \\
\hline Bisaccate & - & - & - & - & - & & - & & 1 & - & - & - & - & - \\
\hline Inaperturate & - & - & - & - & - & & - & & - & - & 1 & - & - & - \\
\hline Cedrus & - & - & 1 & - & - & & - & & - & - & - & - & 1 & - \\
\hline Keteleeria & - & - & - & - & - & & - & & - & - & - & - & - & - \\
\hline Picea-Abies & - & - & - & 1 & 2 & & 11 & & 1 & 6 & 2 & 4 & - & - \\
\hline Pinus & 13 & - & 50 & 17 & 7 & & 9 & & - & 20 & 10 & 20 & 27 & 6 \\
\hline Podocarpus & - & - & - & - & - & & - & & - & - & - & - & - & - \\
\hline Sciadopitys & - & - & - & - & - & & - & & - & - & - & - & - & - \\
\hline Sequoia & - & - & 3 & - & - & & - & & - & - & - & - & - & - \\
\hline Taxodiad & - & - & - & 1 & - & & - & & - & - & - & - & - & - \\
\hline Tsuga & - & - & - & - & - & & - & & - & - & - & - & - & - \\
\hline Vitreisporites & - & - & - & - & - & & - & & - & - & - & - & - & - \\
\hline Corollina & - & - & - & - & - & & - & & - & - & - & - & - & - \\
\hline Ephedra & - & - & 1 & 1 & - & & 1 & & - & - & - & - & - & - \\
\hline Monocots & & & & & & & & & & & & & & \\
\hline Gramineae & - & - & 12 & 1 & 5 & & 4 & & 2 & - & 22 & 8 & - & 5 \\
\hline Typha & - & - & - & - & - & & 1 & & - & 1 & - & - & - & - \\
\hline Dicots & & & & & & & & & & & & & & \\
\hline Alnus & - & - & 3 & 2 & 2 & & - & & - & 1 & 1 & 1 & 1 & 3 \\
\hline Amaranthaceae & - & - & - & - & - & & - & & - & - & - & - & - & - \\
\hline Betula & - & - & 2 & 2 & 2 & & 1 & & 3 & 3 & 1 & - & - & 1 \\
\hline Carpinus - Ostrya & - & - & 3 & - & 2 & & - & & - & 2 & - & - & 1 & - \\
\hline Carya & - & 1 & 2 & - & 1 & & 1 & & 1 & 2 & 1 & 3 & 1 & 2 \\
\hline Caryophyllaceae & - & - & - & - & - & & - & & - & - & 1 & 1 & - & - \\
\hline Chenopodiaceae & 5 & 4 & 25 & 4 & - & & 12 & & 1 & - & 5 & 6 & - & - \\
\hline Compositae & & & & & & & & & & & & & & \\
\hline Undifferentiated & 4 & - & 5 & - & - & & 3 & & - & - & - & - & - & - \\
\hline Artemisia & 2 & 1 & 5 & - & 1 & & 4 & & - & - & - & - & 1 & - \\
\hline Long-spined & - & - & - & - & - & & - & & - & - & - & 2 & - & - \\
\hline Fenestrate & - & - & - & - & - & & - & & - & - & 1 & - & - & - \\
\hline Corylus & - & - & - & 3 & - & & - & & - & - & - & - & - & - \\
\hline Engelhardia & - & - & - & - & 6 & & - & & 31 & - & 20 & 13 & 31 & 22 \\
\hline Fagus & - & - & - & - & 1 & & 1 & & - & - & - & - & - & - \\
\hline Hex & - & - & - & - & - & & - & & - & - & - & - & - & - \\
\hline Myrica & - & - & 1 & 1 & - & & - & & - & - & - & - & - & - \\
\hline Pterocarya & - & - & 1 & - & - & & 2 & & - & 2 & - & - & - & - \\
\hline Quercus & 5 & 1 & 10 & 3 & 7 & & 3 & & 26 & 7 & 1 & - & 6 & 8 \\
\hline Schizandra & - & - & - & - & - & & - & & - & - & - & - & - & - \\
\hline Tilia & - & - & - & - & - & & - & & - & 1 & - & - & 1 & 1 \\
\hline Ulmaceae & 4 & 5 & 9 & 2 & 1 & & 11 & & 1 & 4 & - & 2 & 1 & 2 \\
\hline Umbelliferae & 1 & 1 & 2 & - & - & & 1 & & - & - & - & 1 & - & - \\
\hline
\end{tabular}





\section{PLATE 1}

Figures 1-4

Figures 5-9

Figures 15-17

Acritarch-13. Figures 15 and 16 are two levels of focus of the same specimen. This distinctive palynomorph is abundant in a few samples, (i.e., Sample 381-27, CC). Takes a small amount of safranin-o. Sculpture rugulate-reticulate. "Eye-spot" characteristic of many dinoflagellate cysts always present, as well as some hint of an archeopyle (see Figure 15, especially). This may well prove to be another of the Black Sea "baggy" dinoflagellates, but the proof (opercula, tabulation) has not as yet been found. Size: 23 $\mu \mathrm{m}$.

Figures 18-23 Dinoflagellate-24a. Dominant in a few samples (i.e., Sample 381-23, CC). Probably one of the "baggy" dinoflagellates of the Black Sea sediments. At first, this was considered an acritarch, but eventually archeopylar evidence of probable dinoflagellate relationship was discovered (see especially Figure 21). However, no tabulation has been seen. Stains lightly with safranin-o. Very thin walled. More or less psilate, with some granules. Size: about $22 \mu \mathrm{m}$.

Figures 24-27 Dinoflagellate-29. A nondescript, "baggy" dinoflagellate such as typifies the Black Sea sediments. In one sample (Sample $381-38, \mathrm{CC}$ ) this form is overwhelmingly dominant; the organic residue in that sample consists practically $100 \%$ of this dinoflagellate. "Baggy", thin walled, lightly staining, micro-scabrate sculpture. Tabulation never observed. Figure 24 is an isolated operculum. These pre-cingular opercula can be seen in situ in Figures 25-27. Size: about $55 \mu \mathrm{m}$. (Figure 24 is approximately $30 \%$ more magnified than Figures 25-27.)

Figure 28 Dinoflagellate-28. Common in some samples, such as Sample 381-33, CC. Orangebrown color, usually non-staining. Sculpture densely scabroverrucate. Figure shows an operculum torn loose but still partly attached. Some specimens show signs of weakly developed tabulation. Size: about $68 \mu \mathrm{m}$ long. 
PLATE 1
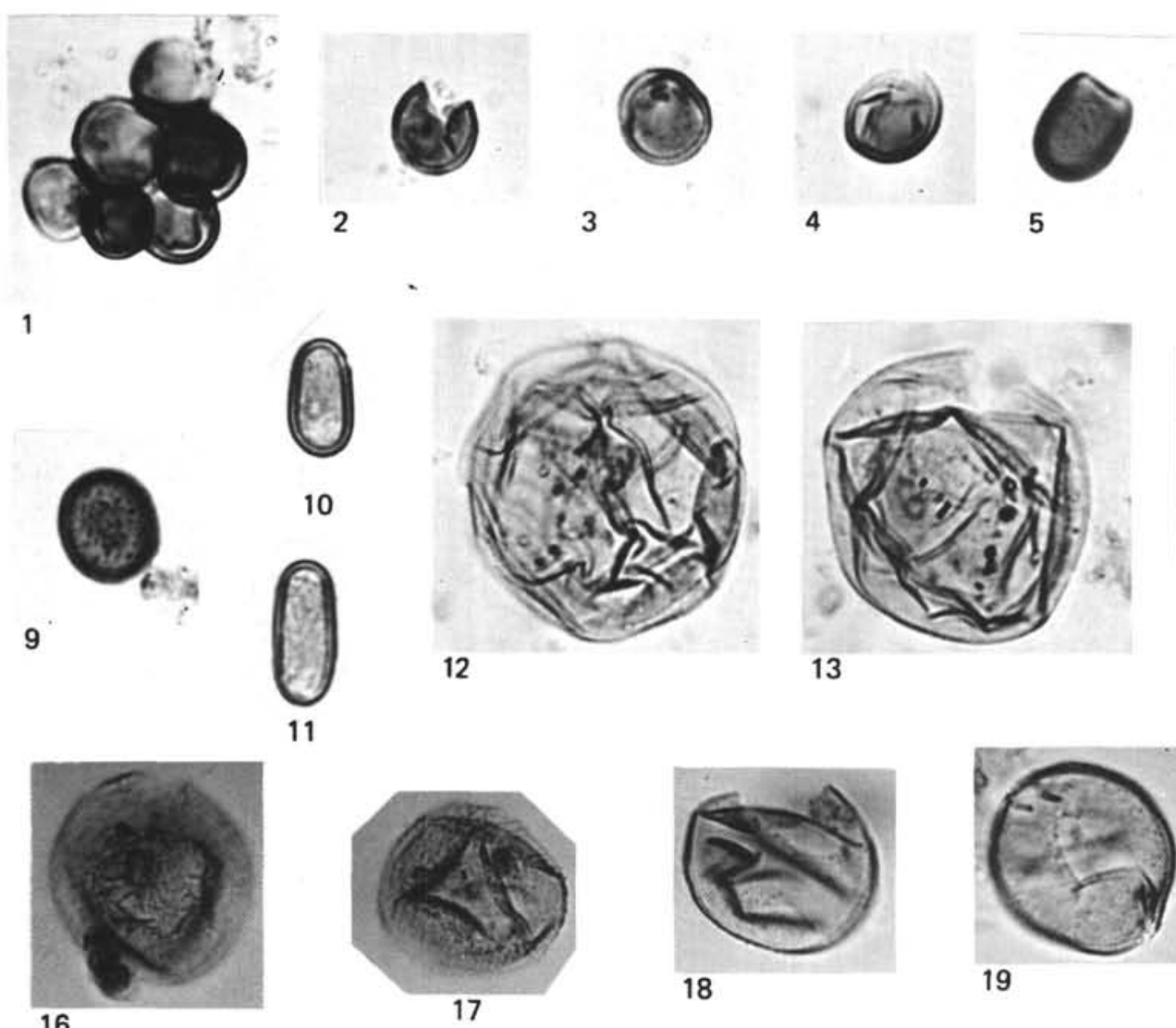

11
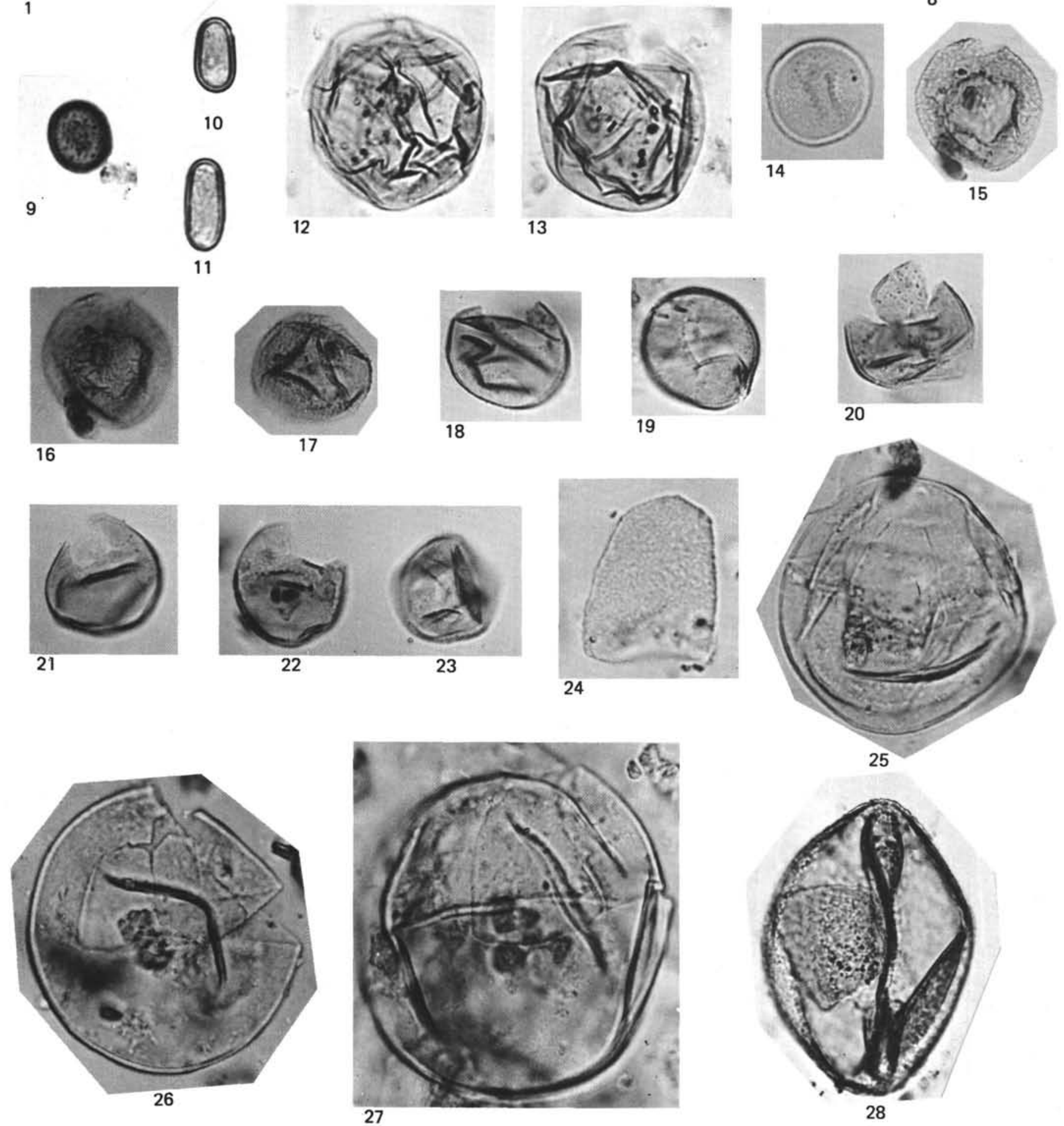
PLATE 2

Figures 1-6,8 Dinoflagellates 19-20. This is one of the most important palynomorphs in the Black Sea sediments. When first encountered, these "bags" were thought to be inaperturate pollen grains, but discovery of good pre-cingular archeopyles and opercula (Figures 2,3) made it obvious that this is a dinoflagellate, though a peculiarly featureless and often contorted one, in which the simple "bags" show no sign of tabulation. Dinoflagellate-19 is virtually psilate (Figures 1, 3), whereas Dinoflagellate-20 is reticulate (Figure 8). The two forms intergrade, however (see Figure 4), and it is probable that the two are really one species. Characteristically, many examples of this dinoflagellate occur as tightly folded specimens that, at first glance, seem unrelated to the expanded forms (see Figures 5 and 8). However, all intergradations between specimens even more tightly folded than that shown in Figure 5, and normally expanded cysts, exist. Dinoflagellates 19 20 apparently are sensitive indicators of some ecologic factor, as they fairly often dominate the planktonic flora. Usually a golden or orange color, taking little or no stain. Size of expanded cysts: about $70 \mu \mathrm{m}$ maximum dimension.

Figures 7,9 Dinoflagellate-26. Another "baggy" dinoflagellate. Microreticulate, with no evidence of tabulation, but (see Figure 7) a characteristic, presumably, pre-cingular, archeopyle is present. Orangish in color, usually non-staining. Variable in size; about $60 \mu \mathrm{m}$ on the average.

Figure 10 Undifferentiated dinoflagellate. This sort of more "normal" dinoflagellate cyst is common in some samples. Verrucate sculpture. Prominent cingulum and rather evident tabulation. Size: about $70 \mu \mathrm{m}$. 
PLATE 2
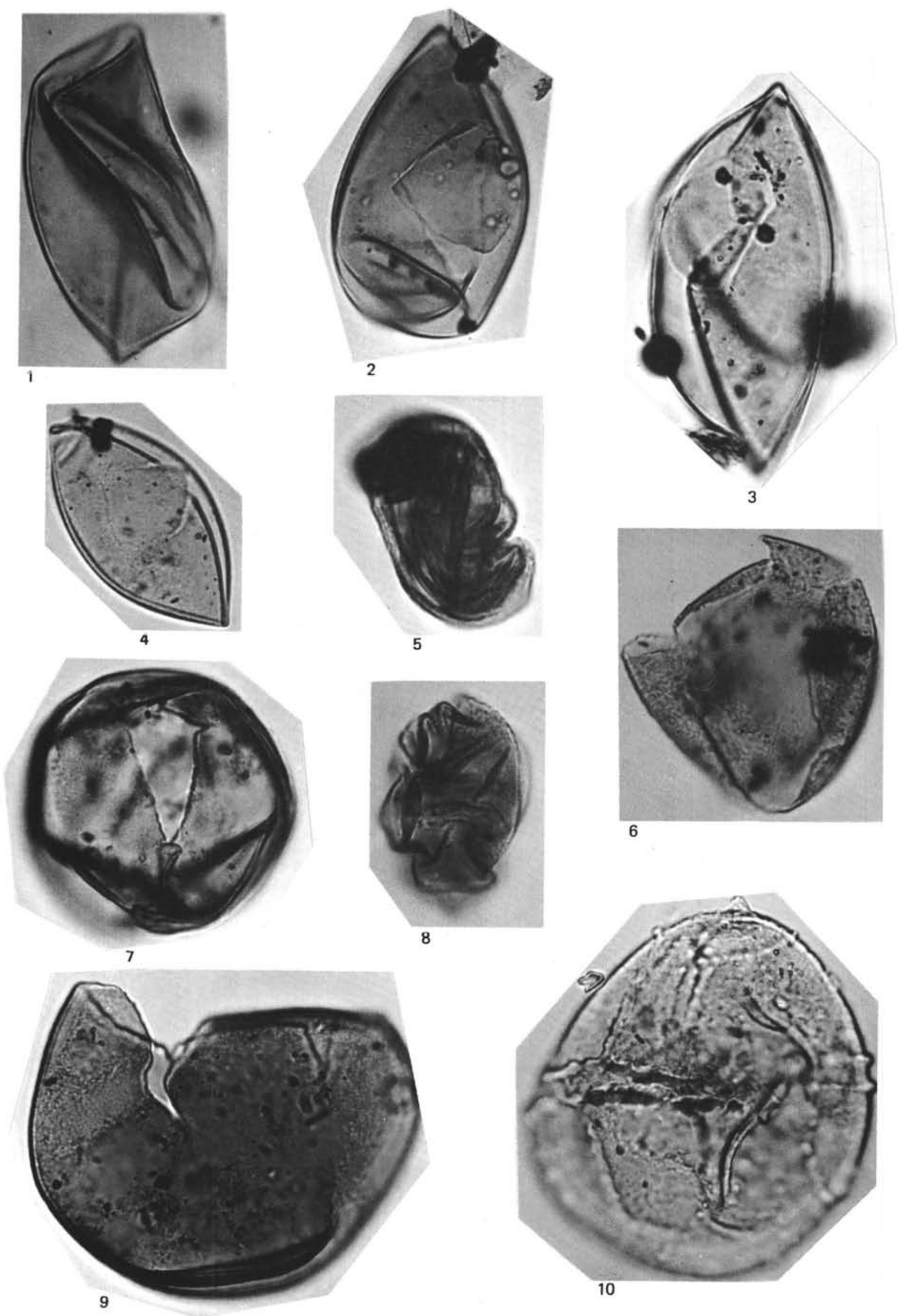